Ferrata Storti Foundation

\title{
PIEZ01 activation delays erythroid differentiation of normal and hereditary xerocytosis-derived human progenitor cells
}

Haematologica 2020

Volume 105(3):610-622

\section{Correspondence:}

LOÏC GARÇON

garcon.loic@chu-amiens.fr

Received: February 11, 2019.

Accepted: August 9, 2019.

Pre-published: August 14, 2019.

doi:10.3324/haematol.2019.218503

Check the online version for the most updated information on this article, online supplements, and information on authorship \& disclosures: www.haematologica.org/content/105/3/610

(C)2020 Ferrata Storti Foundation

Material published in Haematologica is covered by copyright. All rights are reserved to the Ferrata Storti Foundation. Use of published material is allowed under the following terms and conditions:

https://creativecommons.org/licenses/by-nc/4.0/legalcode. Copies of published material are allowed for personal or internal use. Sharing published material for non-commercial purposes is subject to the following conditions:

https://creativecommons.org/licenses/by-nc/4.0/legalcode, sect. 3. Reproducing and sharing published material for commercial purposes is not allowed without permission in writing from the publisher.

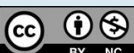

\begin{abstract}
Alexis Caulier,${ }^{1,2^{*}}$ Nicolas Jankovsky, ${ }^{1 *}$ Yohann Demont,${ }^{3}$ Hakim Ouled-Haddou, ${ }^{1}$ Julien Demagny, ${ }^{4}$ Corinne Guitton, ${ }^{5}$ Lavinia Merlusca, ${ }^{2}$ Delphine Lebon, ${ }^{1,2}$ Pascal Vong, ${ }^{1}$ Aurélien Aubry, ${ }^{1}$ Agnès Lahary, ${ }^{6}$ Christian Rose,${ }^{7}$ Sandrine Gréaume, ${ }^{8}$ Emilie Cardon, ${ }^{1}$ Jessica Platon, ${ }^{1}$ Halima Ouadid-Ahidouch, ${ }^{9}$ Jacques Rochette, ${ }^{1,10}$ Jean-Pierre Marolleau, ${ }^{1,2}$ Véronique Picard ${ }^{11}$ and Loïc Garçon ${ }^{1,410}$
\end{abstract}

${ }^{1}$ EA4666 HEMATIM, Université Picardie Jules Verne, Amiens; ${ }^{2}$ Service des Maladies du

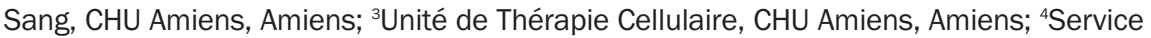
d'Hématologie Biologique, CHU Amiens; ${ }^{5}$ Service de Pédiatrie Générale, CHU Bicêtre, APHP, Le Kremlin-Bicêtre; ' ${ }^{6}$ Laboratoire d'Hématologie, CHU Rouen, Rouen; ' $S e r v i c e$ d'Oncologie et d'Hématologie, Hôpital Saint Vincent de Paul, Lille; ${ }^{8}$ Etablissement Français du Sang (EFS) de Normandie, Bois-Guillaume; ${ }^{9}$ EA4667 Laboratoire de Physiologie Cellulaire et Moléculaire, Université Picardie Jules Verne, Amiens;

${ }^{10}$ Laboratoire de Génétique Moléculaire, CHU Amiens, Amiens and ${ }^{11}$ Laboratoire d'Hématologie, AP-HP, Le Kremlin-Bicêtre, France

${ }^{\star} A C$ and $N J$ contributed equally to this work.

\section{ABSTRACT}

H ereditary xerocytosis is a dominantly inherited red cell membrane disorder caused in most cases by gain-of-function mutations in PIEZO1, encoding a mechanosensitive ion channel that translates a mechanic stimulus into calcium influx. We found that PIEZO1 was expressed early in erythroid progenitor cells, and investigated whether it could be involved in erythropoiesis, besides having a role in the homeostasis of mature red cell hydration. In UT7 cells, chemical PIEZO1 activation using YODA1 repressed glycophorin A expression by $75 \%$. This effect was PIEZO1-dependent since it was reverted using specific short hairpin-RNA knockdown. The effect of PIEZO1 activation was confirmed in human primary progenitor cells, maintaining cells at an immature stage for longer and modifying the transcriptional balance in favor of genes associated with early erythropoiesis, as shown by a high GATA2/GATA1 ratio and decreased $\alpha / \beta$ globin expression. The cell proliferation rate was also reduced, with accumulation of cells in G0/G1 of the cell cycle. The PIEZO1-mediated effect on UT7 cells required calcium-dependent activation of the NFAT and ERK1/2 pathways. In primary erythroid cells, PIEZO1 activation synergized with erythropoietin to activate STAT5 and ERK, indicating that it may modulate signaling pathways downstream of erythropoietin receptor activation. Finally, we studied the in-vitro erythroid differentiation of primary cells obtained from 14 PIEZO1-mutated patients, from 11 families, carrying ten different mutations. We observed a delay in erythroid differentiation in all cases, ranging from mild $(n=3)$ to marked $(n=8)$. Overall, these data demonstrate a role for PIEZO1 during erythropoiesis, since activation of PIEZO1 both chemically and through activating mutations - delays erythroid maturation, providing new insights into the pathophysiology of hereditary xerocytosis.

\section{Introduction}

PIEZO proteins were identified as a family of mechanically activated transductors, first described in neuronal cell lines, which convert mechanical forces into biological signals. ${ }^{1}$ PIEZO1 is a large, three-blade propeller-shaped protein displaying 38 transmembrane-helix domains, and is encoded by the broadly 
expressed FAM38A gene ${ }^{2,3}$ (GTEx Project) (https://gtexportal.org/home/gene/PIEZO1). Activation of the mechanosensitive ion channel PIEZO1 has a significant effect on red blood cell physiology. Under physiological conditions, PIEZO1 regulates ATP release from human mature erythrocytes, ${ }^{4}$ and has been shown to control red blood cell volume and hydration homeostasis through ion balance. ${ }^{5}$ PIEZO1 induces a cationic current that develops quickly after activation of the protein by a mechanical stress; this activation is followed by a rapid inactivation determined by the C-terminal extracellular domain and the inner pore helix. ${ }^{6}$ PIEZO1 is a non-selective channel although it presents a preferential conductance of monovalent cations, and a significant permeability to calcium $\left(\mathrm{Ca}^{2+}\right){ }^{1,7}$ PIEZO1 gain-of-function mutations have been associated with most cases of hereditary xerocytosis (HX), leading to either a slower inactivation or altered channel kinetics..$^{8-11}$ These mutations induce excessive $\mathrm{Ca}^{2+}$ influx and secondary activation of the Gardos channel in red cells, thereby causing potassium $\left(\mathrm{K}^{+}\right)$leakage, water loss, and erythrocyte dehydration..$^{12,13}$

So far, the role of PIEZO1 during erythropoiesis has only been described in mature erythrocytes. However, it is also expressed earlier in human erythroid progenitors. ${ }^{8,14}$ In many cell types such as epithelial, urothelial and endothelial cells, PIEZO1 has been involved in regulation of the cell cycle, proliferation and differentiation. ${ }^{15-}$ ${ }^{18}$ Prompted by a recent report that a PIEZO1 mutation could mimic myelodysplastic syndrome with megaloblastic features,$^{19}$ we performed an extensive and comprehensive investigation of PIEZO1 expression and function using primary human erythroid progenitor cells. We investigated consequences of its activation either by the selective activator YODA1 in normal human erythroid progenitors or by activating mutations in HX-derived hematopoietic progenitors from 14 patients carrying ten different mutations. We observed that PIEZO1 activation in our models modified the kinetics of erythropoiesis, inducing a delay in terminal erythroid differentiation. Our results suggest that PIEZO1 plays a key role during human erythroid differentiation.

\section{Methods}

The primary cell culture protocol, multiparametric flow cytometry (MFC), live imaging flow cytometry (IFC), western blot, immunofluorescence, quantitative reverse transcriptase polymerase chain reaction (RT-qPCR) analysis and reagents are detailed in the Online Supplementary Methods.

\section{Cell lines culture}

The UT7 cell line was maintained in $\alpha$-minimum essential medium (Dominique Dutscher) supplemented with 10\% fetal calf serum (Eurobio) and cytokines. Two subclones were used: the UT7/GM clone and the UT7/EPO clone. The UT7/GM clone was used as a model of erythropoietin (EPO)-driven differentiation, proliferating under $5 \mathrm{ng} / \mathrm{mL}$ recombinant human granulocyte-macrophage colony-stimulating factor (GM-CSF; Miltenyi) and differentiating under $5 \mathrm{U} / \mathrm{mL}$ EPO after GM-CSF had been removed by two washes in $1 \mathrm{x}$ phosphate-buffered saline..$^{20}$ The UT7/EPO clone (a gift from Dr. Y.Zermati, Institut Cochin, Paris, France) was used as a model of EPO-driven proliferation, and cultured with $2 \mathrm{U} / \mathrm{mL}$ EPO (Online Supplementary Figure S1). ${ }^{21}$

\section{Patients' samples and primary cell cultures}

HX hematopoietic cells were obtained from blood samples collected into EDTA or from phlebotomy bags after patients' informed consent in accordance with the Helsinki declaration. This study was conducted in compliance with French legislation on non-interventional studies. Patients' data were collected directly from their medical records or through the HX French Cohort registry, approved by the National Commission on Informatics and Liberty. The diagnosis of HX was based on clinical and biological features and a typical osmotic gradient ektacytometry curve before genetic testing. Thirteen of the patients in this study were recently extensively described. ${ }^{22}$ Control samples were obtained from blood or mobilized peripheral blood mononuclear cells (MNC) of healthy subjects. The MNC were isolated using density gradient-centrifugation (Ficoll-Paque PLUS, GE Healthcare) and CD34+ cells were sorted by magnetic microbead separation on MACS columns (AutoMACS Separator). The complete protocol for erythroid differentiation is described in Online Supplementary Figures S1 and S2 and detailed in the Online Supplementary Methods.

\section{Lenti/retroviral production and cell infection}

Four short hairpin (Sh) RNA against PIEZO1 (Sh-PIEZO1) and one control scrambled ShRNA (Sh-SCR) cloned in pLKO.1CMV-tGFP vector were designed using the Mission ${ }^{\circledast}$ shRNA tool and purchased from Sigma-Aldrich (detailed sequences are provided in Online Supplementary Table S1). Specific anti-PIEZO1 targeting was verified using an online alignment research tool (nucleotide BLAST ${ }^{\circledR}, \mathrm{NCBI}$ ). Viral production was ensured in the HEK293T cell line, after transfection using Lipofectamine ${ }^{\circledR}$ LTX with Plus $^{\mathrm{TM}}$ reagent (Thermo Fisher Scientific) in antibiotic-free, high-glucose Dulbecco modified Eagle medium (Dominique Dutscher). Lentiviral supernatant was harvested from day 2 to 4 , and filtered through a $0.45 \mu \mathrm{M}$ polyvinylidene fluoride membrane (Millex-HV $0.45 \mu \mathrm{M} 33 \mathrm{~mm}$, Merck-Millipore) before ultracentrifugation on day $4\left(100,000 \mathrm{~g}\right.$ for $90 \mathrm{~min}$ at $4^{\circ} \mathrm{C}$, Optima L-80XP, Beckman-Coulter). We used a mix of the four ShRNA to knockdown PIEZO1 in the UT7/EPO cell line. Infection was performed overnight with $8 \mu \mathrm{g} / \mathrm{mL}$ polybrene (Sigma-Aldrich). In UT7/EPO cells, $10 \mu \mathrm{L}$ of each supernatant were used to infect $5 \times 10^{5}$ cells, and were sufficient to induce $>90 \%$ GFP, both with the Sh-SCR and Sh-PIEZO1 mix. Fortyeight hours after transduction, cells were washed in $50 \mathrm{~mL} 1 \mathrm{x}$ phosphate-buffered saline and cultured for an additional 3 days in the presence of dimethylsulfoxide (DMSO) or YODA1 before $M F C$ staining. The retroviral MigR vector containing dominantnegative MEK was a generous gift from Prof. S. Giraudier (Hôpital Saint-Louis, Paris, France).

\section{Statistical analysis}

Statistical analyses were performed using two-tailed $P$ values and parametric tests. The $\alpha$ value for statistical significance was set at 0.05 . For quantitative variables we used a Student $t$-test for paired or unpaired samples depending on the experiment under consideration. All numerical values are expressed as mean values \pm standard deviation.

\section{Results}

PIEZ01 is expressed at an early stage during in vitro erythropoiesis of human CD34+ cells

We first assessed PIEZO1 expression during synchronized human in vitro erythroid differentiation as described in Online Supplementary Figure S2B. PIEZO1 mRNA was 
preferentially expressed in $\mathrm{CD} 34^{+}$cells and in early stages of erythropoiesis from day 4 to 10 (corresponding to burst-forming unit-erythroid/colony-forming unit-erythroid/proerythroblast in our culture system) then decreased during terminal maturation (Figure 1A). This was in agreement with previously published RNAsequencing analyses on erythroid precursors. ${ }^{14,23,24}$
Expression of glycophorin A (GPA) was measured in parallel as a positive marker of erythroid differentiation (Figure 1B). Similar results were observed at the protein level using MFC (Figure 1C, D). The specificity of PIEZO1 antibody staining using MFC was verified by western blot and immunofluorescence assays (Online Supplementary Figure S4).
A

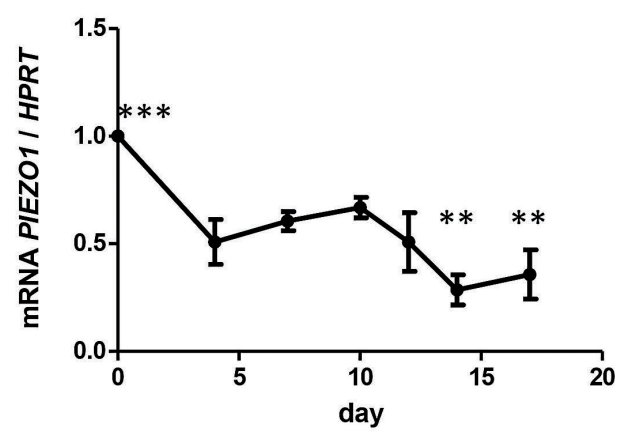

C

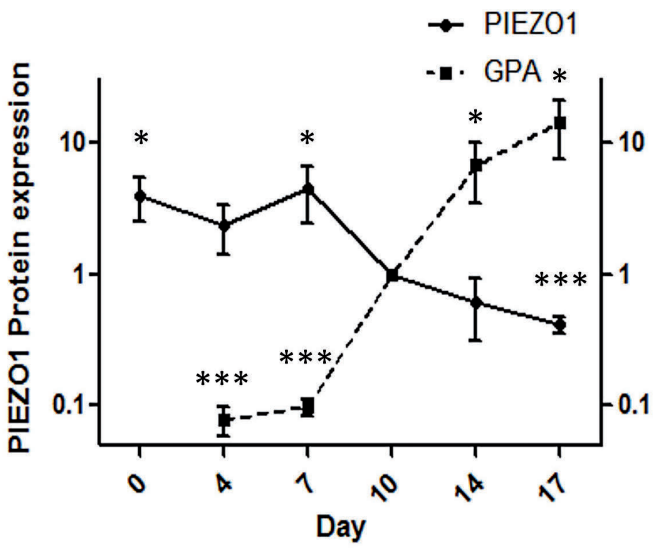

B

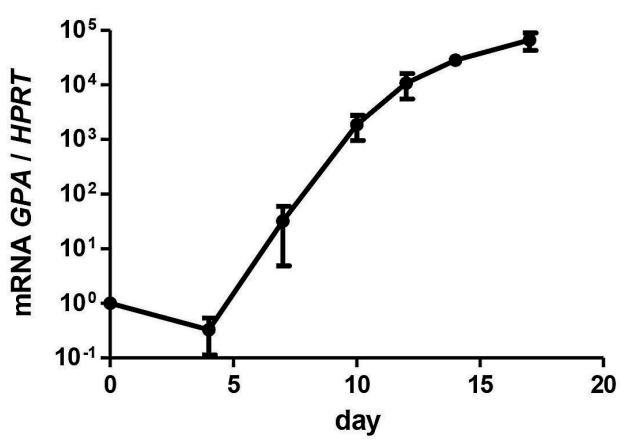

D
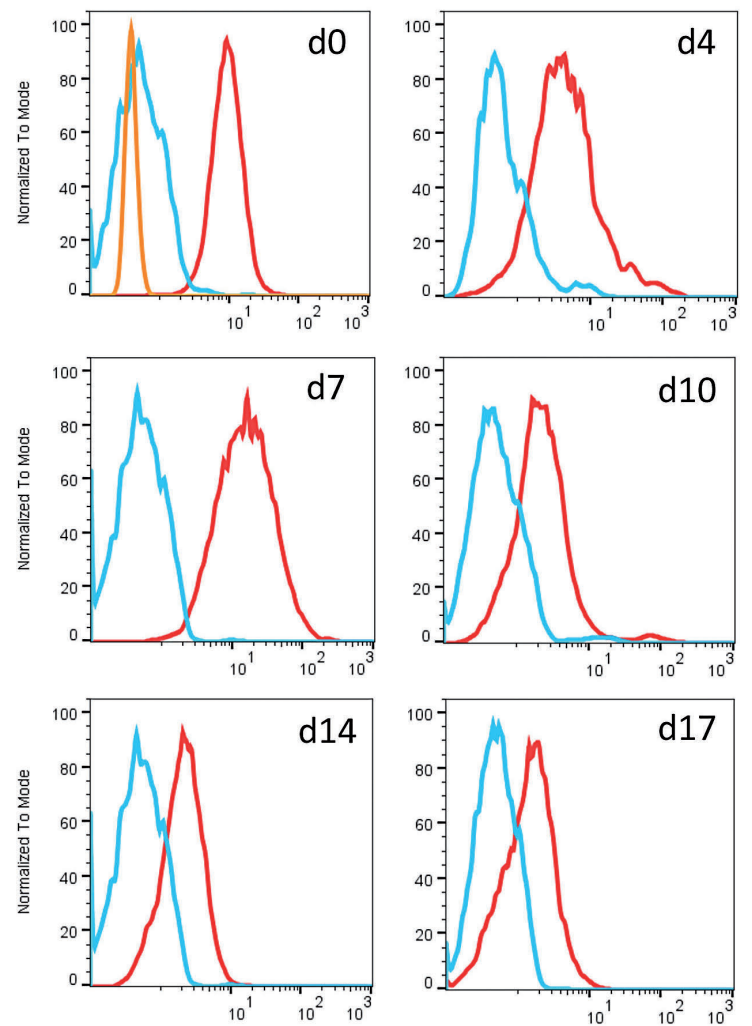

PIEZO1 protein expression

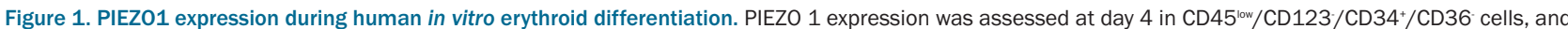
at day 7 in $\mathrm{CD}_{36}{ }^{+}$cells, for both the gene and protein expression experiments. (A) PIEZO1 mRNA expression (determined by quantitative reverse transcriptase polymerase chain reaction, RT-qPCR) relative to HPRT expression, during synchronized erythroid differentiation. Differential expression relative to day 0 . Statistical analysis was made compared to day 10. No significant change was seen at days 4, 7, and 12. (B) Glycophorin A (GPA) mRNA expression (determined by RT-qPCR) relative to HPRT expression, during synchronized erythroid differentiation. Reference was day 0. (C) Kinetics of relative PIEZO1 protein expression during in-vitro erythroid differentiation, in parallel to relative GPA membrane expression. For both, expression at each time point was assessed by multiparametric flow cytometry (MFC) (mean fluorescence intensity at the time point relative to that at day 10.) (D) MFC histograms of PIEZ01 protein expression assessed at different culture time points (red). We used both the secondary antibody alone (blue) and a non-specific rabbit anti HLA-DR1 antibody (orange) as controls. ( $\mathrm{n}=3$ for all experiments) $* * * P<0.001 ; * * P<0.01 ; * P<0.05$ 
YODA1 inhibits cell proliferation and blocks erythroid differentiation in a PIEZ01-dependent manner in UT7 cells

The UT7/GM cell line was used as a model of EPOinduced erythroid differentiation. Indeed, these cells expressed a low level of GPA under exposure to GM-CSF, and acquired GPA in the presence of EPO (Online Supplementary Figure S1A-C). We tested YODA1, a specif- ic PIEZO1 activator, ${ }^{25}$ at increasing concentrations and selected $5 \mu \mathrm{M}$ for further experiments in these cells (Online Supplementary Figure $S 3 A, B)$. At this concentration, YODA1 decreased cell proliferation (Figure 2A) and increased the percentage of cells in the G0/G1 phase (Figure 2B). No difference in cell mortality or apoptosis rate was noted (Online Supplementary Figure S3C, D). Under the differentiation condition using $5 \mathrm{U} / \mathrm{mL}$ EPO,
A

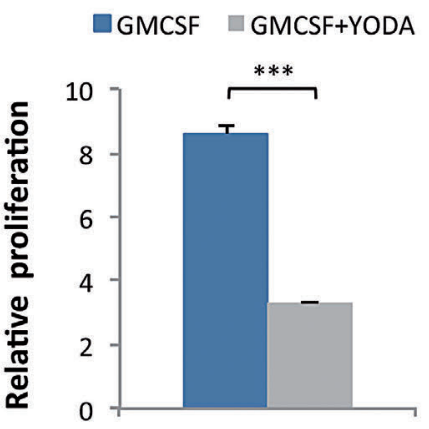

C

aMCSF $\square$ EPO+DMSO $\square$ EPO+YODA

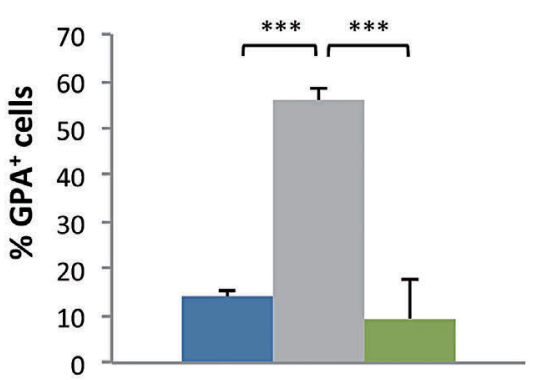

E

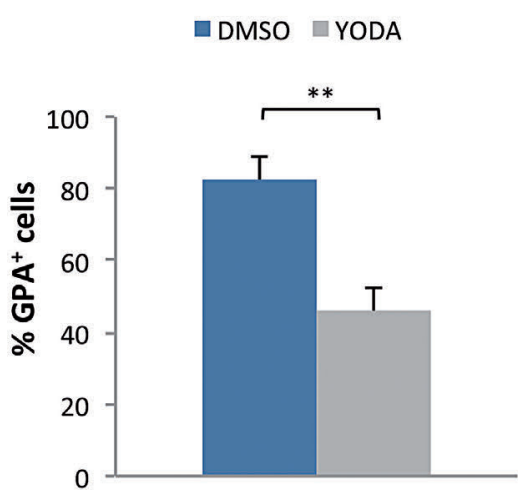

$\square$ GMCSF+DMSO $\square$ GMCSF+YODA

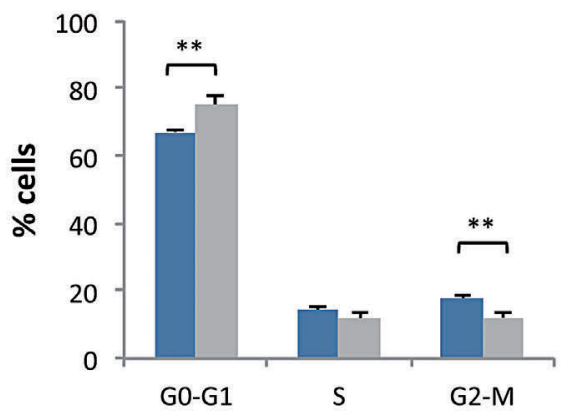

D
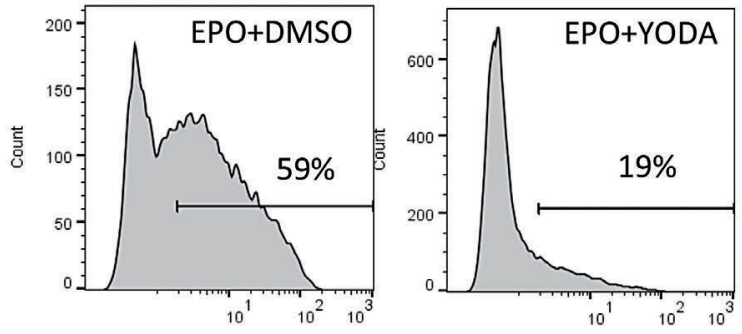

GPA

F

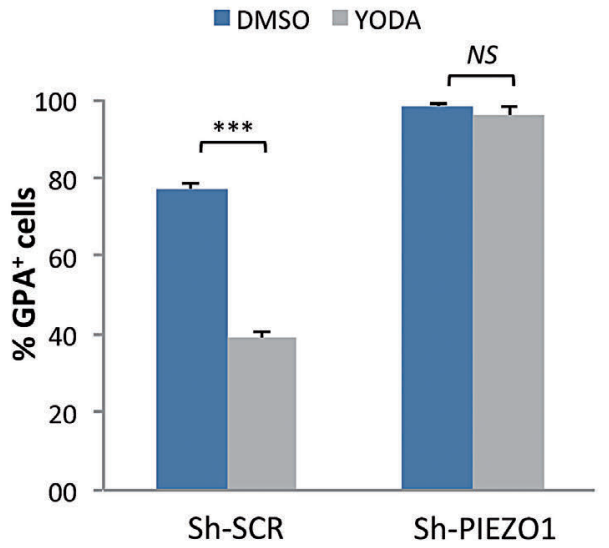

Figure 2. Effect of PIEZO1 chemical activation on the proliferation and differentiation of UT7 cells. Glycophorin A (GPA) expression and cell proliferation were assessed after $72 \mathrm{~h}$ of culture in medium containing granulocyte-macrophage colony-stimulating factor (GMCSF) or erythropoietin (EPO). (A) In the UT7/GM cell line, stimulation with $5 \mu \mathrm{M}$ YODA1 reduced cell expansion compared to that induced by dimethylsulfoxide (DMSO) (2.6 fold). (B) In the UT7/GM cell line exposure to 5 uM YODA1 in GMCSF-containing medium led to cell accumulation in the GO/G1 phase of the cycle $(67 \pm 1 \%$ with DMSO vs. $75 \pm 3 \%$ with YODA1), and a significant decrease in cells in the G2/M phase (18 $\pm 1 \%$ with DMSO vs. $12 \pm 2 \%$ with YODA1). (C) Stimulation with $5 \mathrm{U} / \mathrm{mL}$ EPO induced partial erythroid differentiation in UT7/GM cells, as shown by GPA acquisition ( $56 \pm 3 \%$ vs. $14 \pm 2 \%$ in GMCSF-containing medium), which was strongly inhibited after PIEZO1 chemical activation using $5 \mu \mathrm{M}$ YODA1 ( $9 \pm 8 \%$ with YODA1 vs. $56 \pm 3 \%$ with EPO+DMSO). (D) Representative multiparametric flow cytometry histograms showing the repression of EPO-mediated GPA expression after exposure to YODA1 in UT7/GM cells. (E) GPA repression was also observed in UT7/EPO cells after exposure to 5 uM YODA1 for 3 days ( $82 \pm 6 \%$ vs. $46 \pm 7 \%$ with DMSO). (F) In UT7/EPO cells transduced with Sh-SCR, YODA1 repressed GPA expression (39 $\pm 1.8 \%$ vs. $77 \pm 1.1 \%$ with DMSO) whereas infection with a mixture of four different Sh-PIEZO1 abolished the YODA1-mediated inhibition of GPA ( $96 \pm 2 \%$ with YODA1 vs. $98 \pm 0.4 \%, P=N S)$. ( $n=3$ for all experiments, $* * * P<0.001 ; * * P<0.01)$. 
YODA1 significantly repressed GPA induction (Figure 2C, $\mathrm{D})$, and this effect was dose-dependent (Online Supplementary Figure S3A). Using the UT7/EPO cell line that proliferates in the presence of EPO and expresses a high level of GPA at baseline (Online Supplementary Figure SiA, right), we observed a similar dose-dependent GPA downregulation after exposure to YODA1 (Figure 2E and Online Supplementary Figure S3B, E). We ruled out any YODA1 off-target effect using a Sh-RNA lentiviral strategy that specifically knocks down PIEZO1. Transduction efficiency was $>90 \%$, as assessed by GFP expression. The Sh-RNA-mediated PIEZO1 decrease was $65 \%$ at the RNA level (Online Supplementary Figure S4A). At the protein level, the decrease was $50 \%$ as assessed by MFC (Online Supplementary Figure S4B) in comparison to the level in cells transduced with a Sh-scrambled control (Sh-SCR). The PIEZO1 decrease was confirmed using western blotting and immunofluorescence (Online Supplementary Figure $S 4 C, D)$. Following exposure to YODA1, GPA expression decreased in Sh-SCR-transduced cells but not in cells transduced with Sh-PIEZO1 (Figure 2F). The effect was related to GFP intensity, being weaker in cells expressing Sh-PIEZO1 at a lower level (Online Supplementary Figure S4E). Sh-PIEZO1 had the opposite effect of YODA1 on GPA expression since it enhanced erythroid differentiation by increasing the percentage of $\mathrm{GPA}^{+}$cells compared to the control Sh-SCR. (Figure 2F and Online Supplementary Figure S4E). Sh-PIEZO1 did not revert the decreased proliferation induced by YODA1 exposure, and induced a reduced proliferation rate by itself in comparison to ShSCR (Online Supplementary Figure S4F).

\section{PIEZ01 activation delays erythroid differentiation in human $\mathrm{CD} 4^{+}$-derived erythroid cells}

We then investigated whether the erythroid blockage induced by YODA1 in leukemic cell lines was confirmed in human primary cells. Sorted CD34+ cells were driven into erythroid differentiation (Online Supplementary Figure $S 2 A)$. A dose of $1 \mu \mathrm{M}$ YODA1 was selected for drug exposure based on dose-escalation data (Online Supplementary Figure S5A). Erythroid differentiation was assessed on day 10 by MFC and cytology. YODA1 reduced cell amplification without increasing cell death or apoptosis (Online Supplementary Figure S $5 B-D$ ). Importantly, it led to a drastic delay in GPA acquisition, with accumulation of immature $\mathrm{CD} 36^{+} / \mathrm{CD} 117^{+}$erythroblasts and a decrease in $\mathrm{CD} 71^{+} / \mathrm{GPA}^{\text {High }}$ mature cells (Figure 3A-D). These results were confirmed by cytology, which showed an increase in immature erythroid precursors after exposure to YODA1 (Figure 3E, F). To investigate the timing of the YODA1 effect more precisely, we synchronized the cell culture (Online Supplementary Figure S2B). CD36/GPA colonyforming unit-erythroid cells were sorted on day 7 and exposed sequentially to $1 \mu \mathrm{M}$ YODA1 or DMSO from day 7,10 , or 13 to the end of the culture. Red cell terminal differentiation was evaluated as the mean percentage of enucleated cells on day 21 . The YODA1 effect was maximal when cells were exposed early, from day 7 to 10 , as shown by the significant decrease in the Hoechst negative fraction at day 21 (Online Supplementary Figure S6A, B). A decrease in the ratio of enucleated cells was also observed on cytological analyses after early exposure, with the effect being weaker when YODA1 was administered on day 13 (Online Supplementary Figure S6C). We showed here that erythroid differentiation was delayed but not blocked after PIEZO1 activation, since precursors reached final enucleation, but at a slower rate. Taken together, these data argue for a YODA1 effect at the transition between the GPA ${ }^{\text {low }}$ and $\mathrm{GPA}^{\text {high }}$ stages, corresponding to the beginning of the differentiation of erythroid precursors. ${ }^{26}$

\section{PIEZ01 activation delays erythroid differentiation through transcriptional control}

Using RT-qPCR we investigated whether the YODA1induced delay in erythroid differentiation was driven by global transcriptional control. In human primary erythroid cells, EPO-mediated GPA mRNA induction was inhibited by YODA1, indicating that the GPA repression occurred at the transcriptional level. PIEZO1 activation also reduced EPO-mediated $\alpha$ - and $\beta$-globin mRNA induction (Figure 4A). Since GATA2 expression during erythropoiesis is associated with the early proliferative stage while GATA1 is the key protagonist of terminal differentiation, we evaluated the GATA2/GATA1 mRNA ratio after exposure to YODA1. It increased significantly in comparision with the ratio after exposure to DMSO (Figure 4B). A broader analysis of erythroid gene expression confirmed that primary erythroblasts exposed to YODA1 displayed an immature erythroid profile, with higher expression of BMI1 and STAT5A mRNA and lower ALAS2, EPOR, $A H S P$ and SLC4A1 mRNA expression (Figure 4C).

\section{YODA1-induced inhibition of erythroid differentiation is $\mathrm{Ca}^{2+}$-dependent and does not require activation of the Gardos channel}

PIEZO1-mediated red cell dehydration in $\mathrm{HX}$ is $\mathrm{Ca}^{2+}$ dependent; since PIEZO1 is a non-selective cation channel, we next evaluated whether this was also the case during erythropoiesis. In UT7/GM cells, YODA1 caused a strong dose-dependent increase in cytosolic $\mathrm{Ca}^{2+}$ (Figure $5 \mathrm{~A})$. This effect was abolished in $\mathrm{Ca}^{2+}$-free medium, consistent with YODA1 causing a strong $\mathrm{Ca}^{2+}$ influx from the extracellular to the intracellular compartment (Figure 5A, B). $\mathrm{Ca}^{2+}$ chelation using $2 \mathrm{mM}$ ethylene glycol tetra-acetic acid (EGTA) totally blocked the YODA1-mediated decrease of GPA; this was rescued by addition of $2 \mathrm{mM}$ calcium chloride (Figure 5C). The same effect was observed after intracellular $\mathrm{Ca}^{2+}$ chelation using BAPTAAM (data not shown). Since red cell dehydration in PIEZO1$\mathrm{HX}$ involves a $\mathrm{Ca}^{2+}$-dependent secondary activation of the Gardos channel, ${ }^{20}$ we investigated whether the effect of PIEZO1 on erythroblasts was also Gardos-dependent. As shown in Figure 5D, in UT7/GM cells, Senicapoc, a selective Gardos channel inhibitor, did not revert the YODA1associated blockade of GPA, indicating that YODA1induced inhibition of erythroid differentiation did not require secondary activation of the Gardos channel.

\section{PIEZ01 effect on erythroid differentiation involves modulation of NFAT, ERK1/2, and STAT5 pathways in UT7 cells and human primary erythroblasts}

We next investigated $\mathrm{Ca}^{2+}$-dependent signaling pathways activated downstream PIEZO1 in erythroid cells. Since NFAT is a well-known target of $\mathrm{Ca}^{2+}$-signaling in many cell types ${ }^{27}$ we evaluated whether it was involved in erythroid cells. Calcineurin inhibition using $5 \mu \mathrm{M}$ tacrolimus completely blocked the YODA1-induced GPA repression in UT7/EPO cells (Figure 6A). Moreover, exposure to YODA1 led to substantial nuclear translocation of NFATc1, significantly increasing the similarity score value 
compared to DMSO (Figure 6B, C, and Online Supplementary Figure S7A). These data argue for a role of the PIEZO1/Ca ${ }^{2+} /$ NFAT pathway in the control of erythroid differentiation. We also tested whether the main transduction pathways associated with EPO signaling were involved. We used a strategy based on: (i) protein phosphorylation measurements after stimulation with YODA1 and/or cytokines in cell lines and primary cells and (ii) chemical inhibitors, assuming that inhibition of any pathway downstream of PIEZO1 would revert the YODA1-mediated phenotype. We tested two main pathways: ERK1/2 and STAT5. The phospho-ERK inhibitor
A

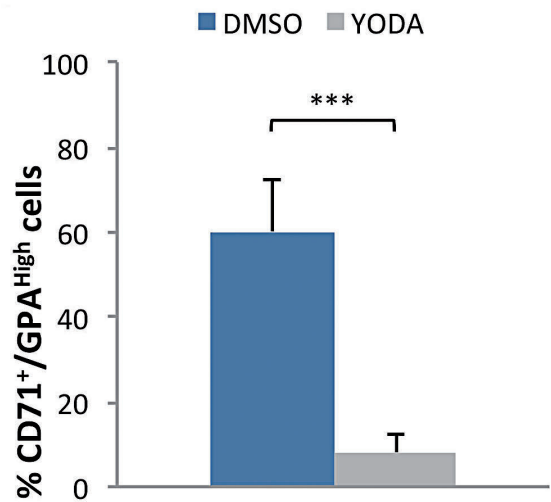

C

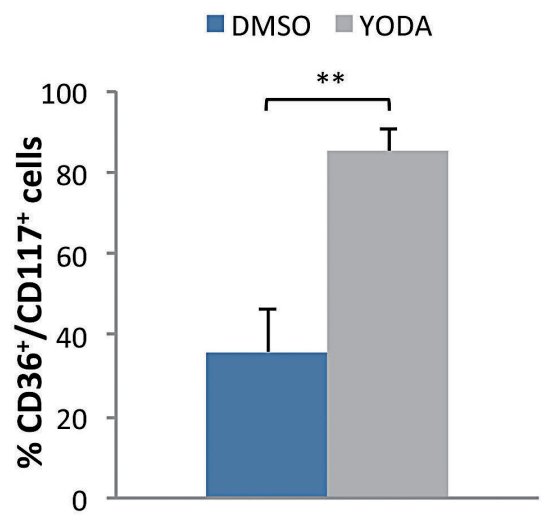

E

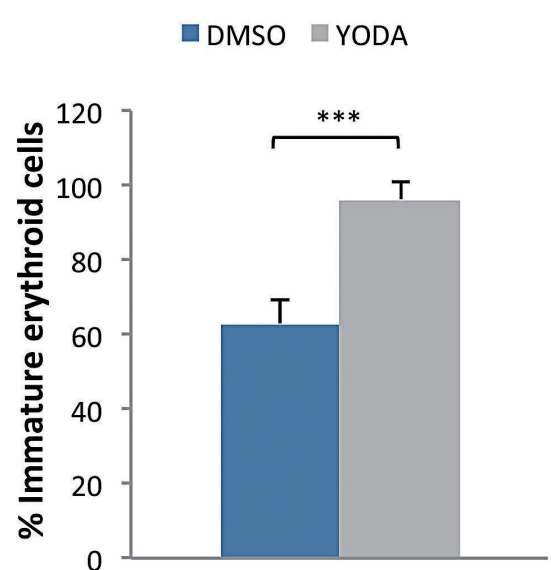

B

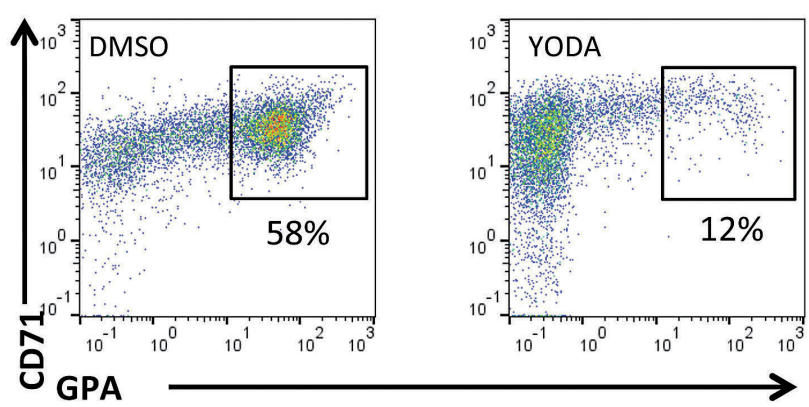

D

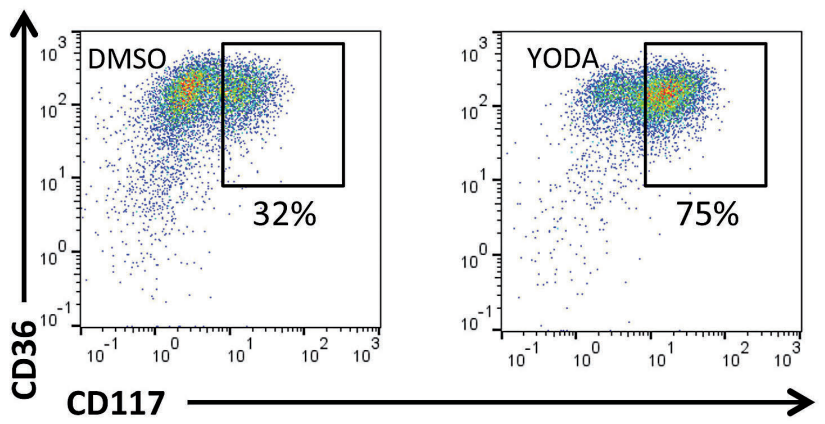

$\mathbf{F}$

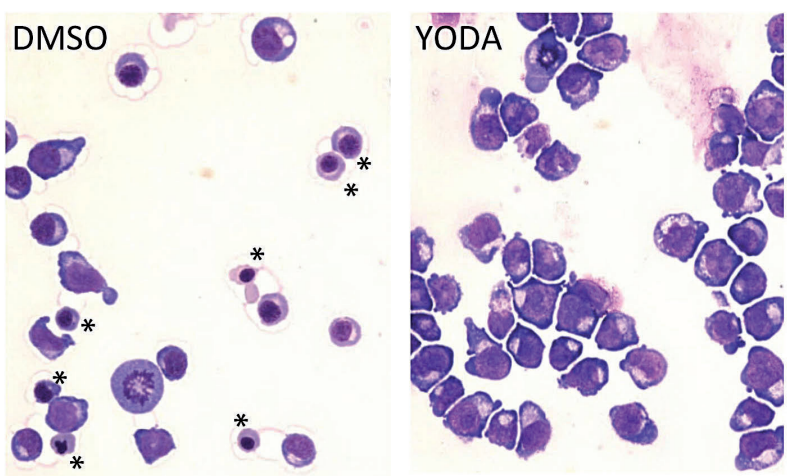

Figure 3. PIEZO1 chemical activation delayed erythroid differentiation of human primary CD34+-derived cells. (A) Exposure to $1 \mu \mathrm{M}$ YODA1 for 3 days decreased the mature erythroblastic population expressing CD71 and GPA ${ }^{\text {High }}[8 \pm 4 \%$ vs. $60 \pm 12 \%$ with dimethylsulfoxide (DMSO)]: multiparametric flow cytometry (MFC) at day 10. (B) Representative MFC plots showing the decrease in the CD71 ${ }^{+}$GPA ${ }^{\text {High }}$ population due to YODA1 (right) compared to the effect of DMSO (left), at day 10 . (C) Exposure to $1 \mathrm{uM}$ YODA1 increased the immature erythroblastic population expressing CD36 and CD117 (85 $\pm 5 \%$ ) compared to that following exposure to DMSO $(36 \pm 11 \%)$. (D) Representative MFC plots showing the increase in CD36 $/$ CD $117^{+}$population due to YODA1 (right) compared to that due to DMSO (left), at day 10 . (E) Excess of immature erythroid cells, i.e., proerythroblasts and basophilic erythroblastsb upon exposure to YODA1 compared to exposure to DMSO, assessed by

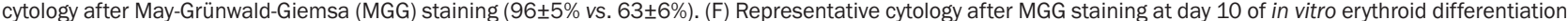
showing a heterogeneous population of erythroblasts at all stages of maturation including the orthrochromatic (*) stage in the control (left) compared to a more homogeneous population of immature erythroblasts in the presence of $1 \mu \mathrm{M}$ YODA (right). ( $\mathrm{n}=4$ in experiment $E, n=3$ in all other experiments). $* * * P<0.001$; $* * P<0.01 ; * P<0.05$. 
UO126 induced spontaneous GPA expression in the absence of EPO, showing that ERK activation was necessary to maintain UT7/GM cells in an undifferentiated $\mathrm{GPA}^{\text {low }}$ state. YODA1 did not revert the high GPA expression induced by ERK1/2 inactivation (Figure 6D). The same results were observed using a retrovirus containing a MEK dominant-negative form (Online Supplementary Figure $S 7 B)$. This indicated that the effect of YODA1 required a functional ERK pathway. Phospho-Flow experiments showed that YODA1 induced strong ERK phosphorylation in UT7/GM cells (Figure 6E). This was confirmed by western blot analysis (data not shown). ERK phosphorylation was $\mathrm{Ca}^{2+}$-dependent, since it decreased strongly in the presence of EGTA (Figure 6E), and PIEZO1-dependent, since it was abrogated in cells transduced with Sh-PIEZO1 lentivirus (Figure 6F). In human primary erythroid progenitors, no $\mathrm{p}$-ERK1/2 was detected in the absence of EPO. In the presence of EPO, YODA1 synergized with EPO for ERK phosphorylation, consistent with a role for PIEZO1 in the modulation of EPO-dependent ERK signaling in primary cells (Figure 6G). This effect was confirmed by western blot analysis (Online Supplementary Figure S7C). We observed a similar synergistic effect in EPO-induced STAT5 phosphorylation in primary erythroid cells (Figure $6 \mathrm{H}$ ), also confirmed by western blotting (Online Supplementary Figure S7D). Of note, no effect of YODA1 on STAT5 phosphorylation was seen in UT7/GM cells, in which STAT5-phopshorylation is not EPO-dependent (Online Supplementary Figure S7E). Taken together, these data argue for a role of PIEZO1 in modulating ERK and STAT5 signaling pathways downstream of EPO-receptor activation in human progenitor cells.

PIEZ01 gain-of-function mutations in hereditary xerocytosis delay erythroid differentiation and mimic the effect of chemical activation

Since most HX patients have PIEZO1 gain-of-function mutations, we tested whether the same phenotype could be observed during in vitro erythroid differentiation from PIEZO1-HX progenitors. Fourteen patients from 11 families (HX \#1 to \#11) carrying ten different PIEZO1 mutations were tested (HX\#3: 2 siblings; HX\#10: 3 siblings). The patients' characteristics are shown in Table 1. Nine phlebotomy samples from five patients [3 families, HX\#1
A

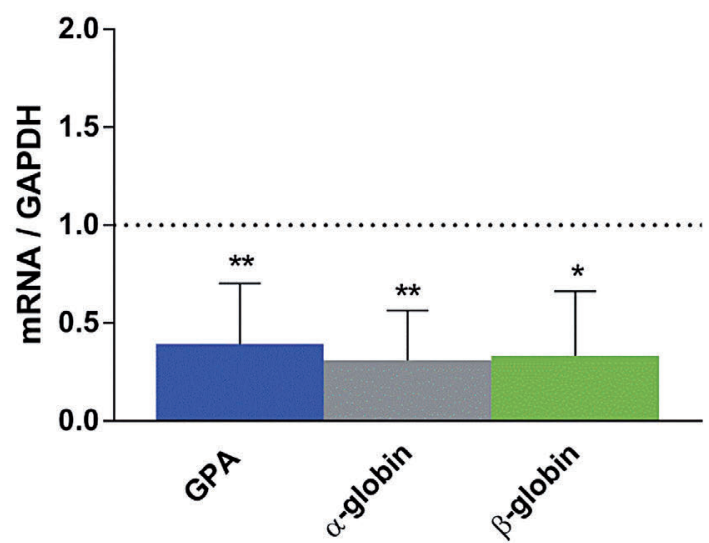

C

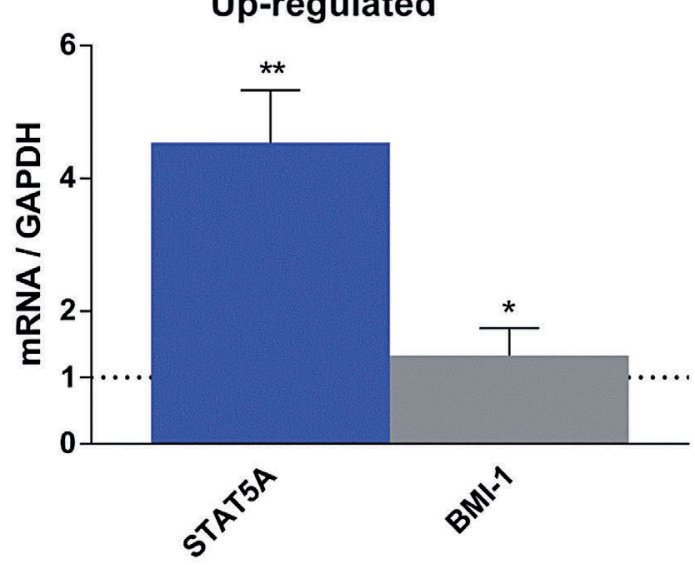

B

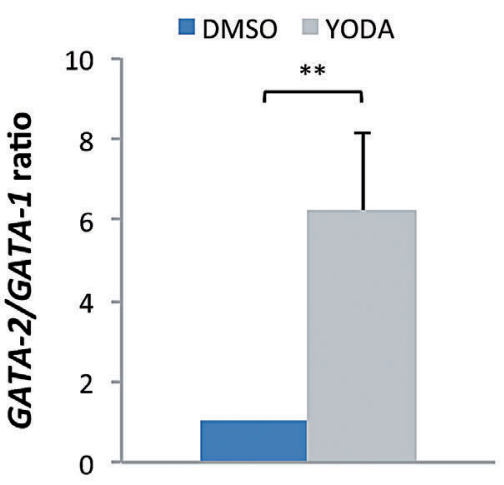

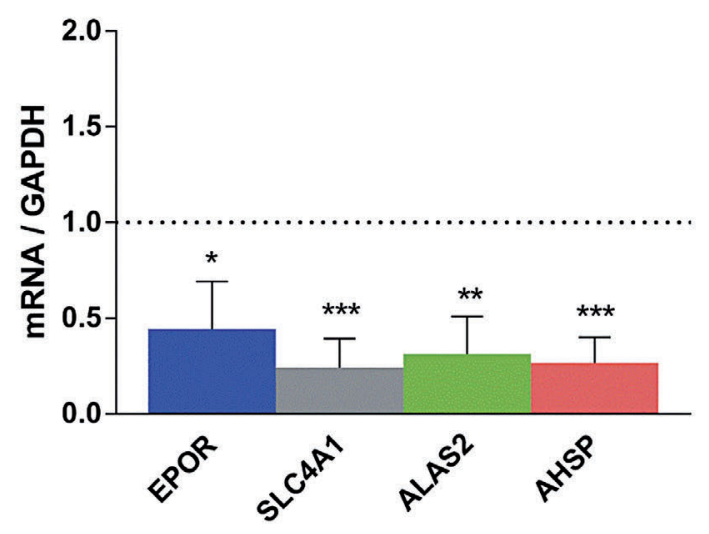

Figure 4. Effect of PIEZO1 activation on the transcriptional program of erythroid differentiation in primary human CD34+-derived cells, assessed by quantitative reverse transcriptase polymerase chain reaction. For all experiments, primary cells were cultured for 10 days, with $1 \mu \mathrm{M}$ YODA1 or dimethylsulfoxide (DMSO) stimulation from day 3 to 10 . Gene expression was assessed relative to GAPDH expression. (A) Compared to exposure to DMSO, exposure to $1 \mathrm{uM}$ YODA1 decreased GPA mRNA expression (x0.49 \pm 0.17 ), $\beta$-globin RNA expression (x0.4 \pm 0.26 ) and $\alpha$-globin RNA expression (x0.3 \pm 0.24$)$. (B) Compared to exposure to DMSO, exposure to 1 $\mu \mathrm{M}$ YODA1 increased the GATA2/GATA1 mRNA ratio (x6.2 \pm 1.9$)$. (C) Stimulation with $1 \mu \mathrm{M}$ YODA1 increased STAT5A and BMI-1 expression, and decreased EPOR, SLC4A1, ALAS2, and AHSP mRNA expression. ( $\mathrm{n}=3$ for all experiments); $* * * P<0.001 ; * * P<0.01 ; * P<0.05$. 
$(n=3), H X \# 2(n=3)$, and HX\#10(n=3)] were used; CD34+ cells were sorted and cultured in erythroid medium. Alternatively, for nine non-phlebotomized patients, MNC were purified from 12 blood samples and were grown directly in the same medium. For controls, we used magnetically sorted $\mathrm{CD}^{+} 4^{+}$cells from mobilized peripheral blood MNC ( $\mathrm{n}=5)$, and $\mathrm{MNC}$ from healthy control blood samples $(\mathrm{n}=9)$. At day 10, we observed a clear delay in erythroid differentiation for seven of the ten PIEZO1-HX mutations (8/14 patients), as shown by a decrease in mature $\mathrm{CD}^{+} 1^{+} / \mathrm{GPA}^{\text {High }}$ cells (Figure $7 \mathrm{~A}$ from sorted CD34 ${ }^{+}$ cells and $7 \mathrm{~B}$ from $\mathrm{MNC}$ ). A moderate but visible delay was observed for the three other PIEZO1-HX mutations (6/14 patients). The intensity of the phenotype was heterogeneous from one mutation to another (Figure 7B), but was reproducible for a given mutation (Figure 7A). For one patient (HX\#1), MNC and CD34+ cells were cultured in parallel and a similar phenotype was observed (one representative dot plot is shown Figure 7C). Cytological analyses after staining with May-Grünwald-Giemsa performed in triplicate for this patient confirmed the delayed erythroid differentiation, showing accumulation of immature erythroblasts (Figure 7D, E). MFC data for all HX mutations and controls are shown in Online Supplementary
A

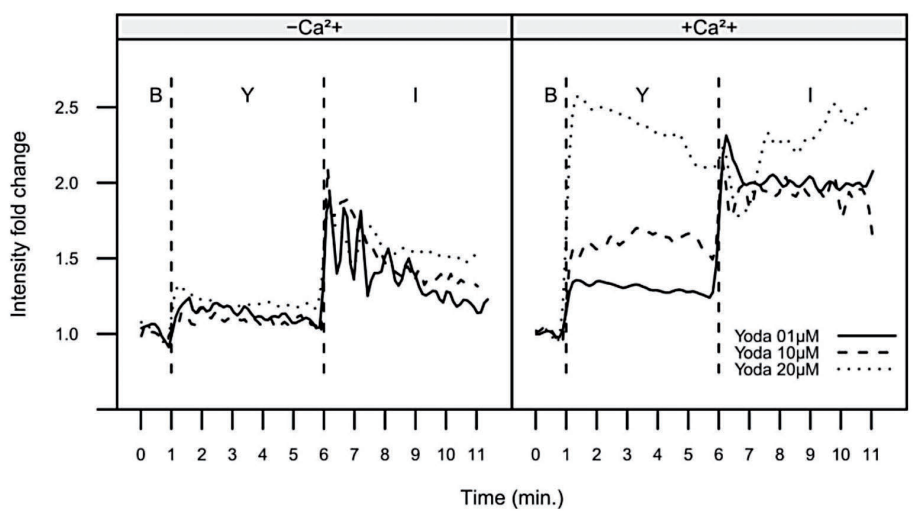

B

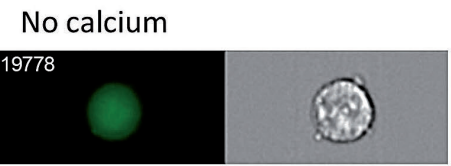

With calcium

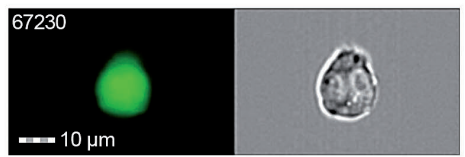

C

D

$\begin{array}{ll}\square \text { DMSO } & \text { YODA } \\ \square \text { YODA+EGTA } & \square \text { YODA+EGTA+CaCl}\end{array}$

$\square$ GMCSF

EPO+DMSO
EPO+YODA

$\square$ EPO+YODA+SENICAPOC
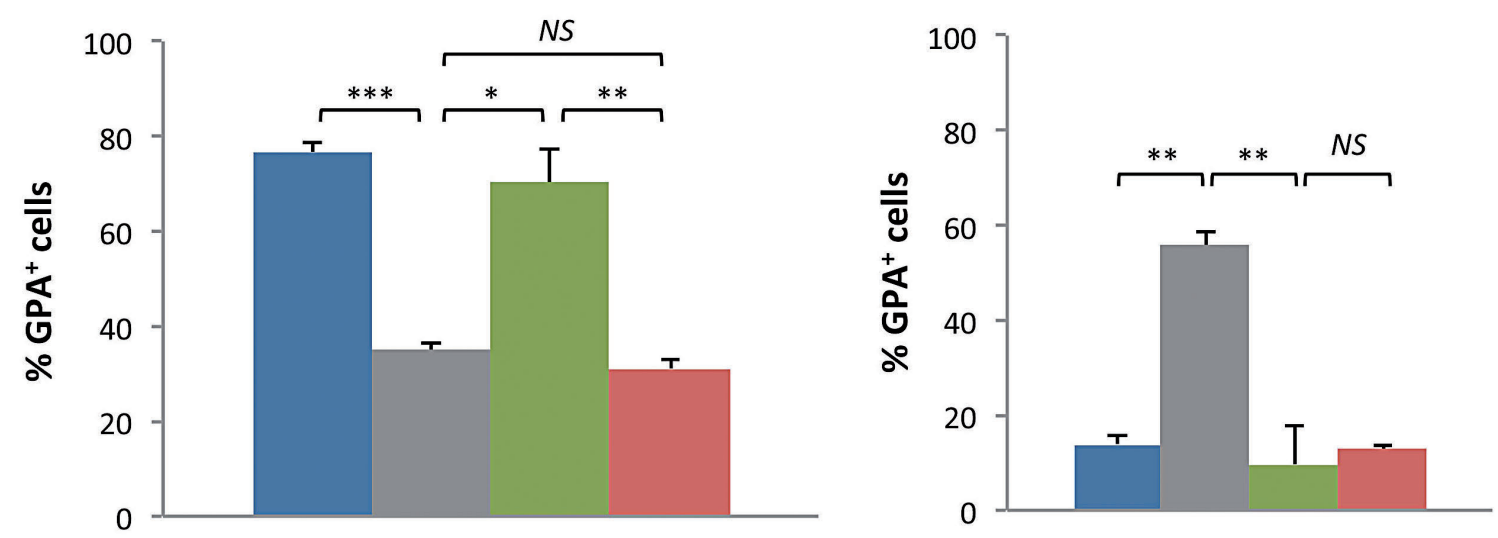

Figure 5. The effect of PIEZO1 activation on erythroid differentiation is calcium-dependent but does not involve a secondary activation of the Gardos channel. (A and B) Cells were incubated with Fluo4-AM for 30 min before stimulation with $5 \mu \mathrm{M}$ YODA1. (C and D) UT7/EPO cells were cultured for $72 \mathrm{~h}$ after drug stimulation. (A) YODA1 stimulation caused a dose-dependent increase in cytosolic calcium concentration in a calcium-containing medium (+Ca ${ }^{2+}$, right panel). No effect was seen in a calcium-free medium (-Ca ${ }^{2+}$, left panel). YODA1 stimulation ("Y") was performed $60 \mathrm{~s}$ after the baseline recording ("B"), before recording for $300 \mathrm{~s}$. The YODA1 concentration was $1 \mu \mathrm{M}$ (solid line), $10 \mu \mathrm{M}$ (dashed line), or $20 \mu \mathrm{M}$ (dotted line). The positive control for an intracellular Ca ${ }^{2+}$ increase was stimulation with $1 \mu \mathrm{M}$ ionomycin (“I") recording for $300 \mathrm{~s}$. The image shown here is representative of three identical experiments. (B) Image of intracellular Ca ${ }^{2+}$ content assessed by ImageStreamX using Fluo4-AM cell permeant, after stimulation with 20 uM YODA1 in $\mathrm{Ca}^{2+}$-containing (lower panel) or $\mathrm{Ca}^{2+}$-free (upper panel) medium. (C) In UT7/EPO cells, exposure to $5 \mu \mathrm{M}$ YODA1 decreased glycophorin A (GPA) expression (35 $\pm 1.4 \%$ ) compared to the expression following exposure to dimethylsulfoxide (DMSO) ( $77 \pm 2 \%)$. Extracellular $\mathrm{Ca}^{2+}$ chelation using $2 \mathrm{mM}$ ethylene glycol tetra-acetic acid (EGTA) prevented the GPA decrease due to YODA1 (70 $\pm 7 \%$ ), and the effect was rescued by adding $2 \mathrm{mM}$ extra calcium chloride (31 $\pm 2 \%)$. (D) Co-exposure with $4 \mu \mathrm{M}$ Senicapoc, a selective Gardos channel inhibitor, did not block the GPA decrease $(13 \pm 1 \%)$ due to YODA1 stimulation $(10 \pm 8 \%, P=N S)$ in erythropoietin (EPO)-containing medium, compared to $D M S O(56 \pm 3 \%)$. ( $n=3$ for all experiments; $* \star \star P<0.001$; $* * P<0.01 ; * P<0.05)$. GMCSF: granulocyte-macrophage colony-stimulating factor. 
A

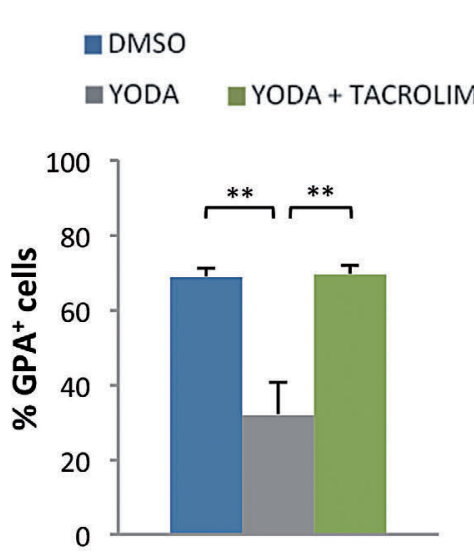

D
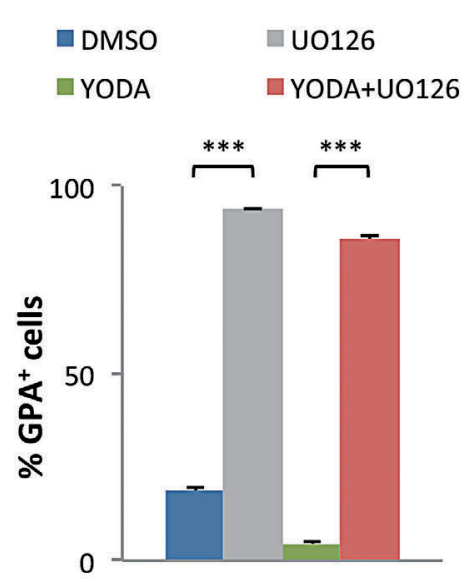

G

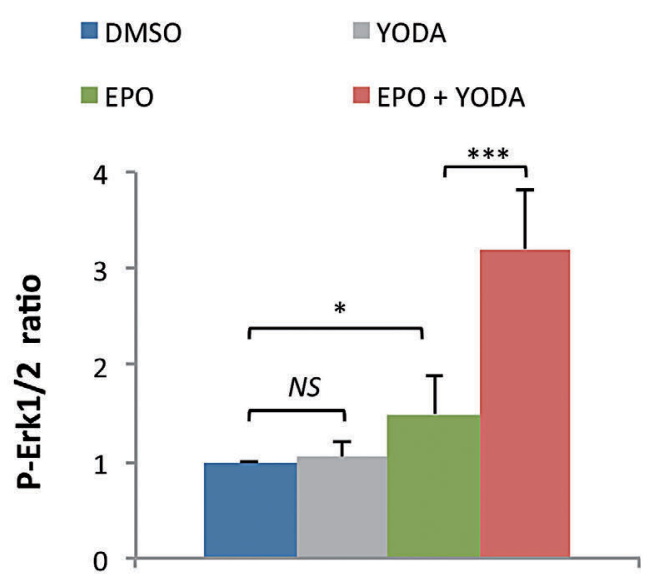

\section{B}

\section{us}

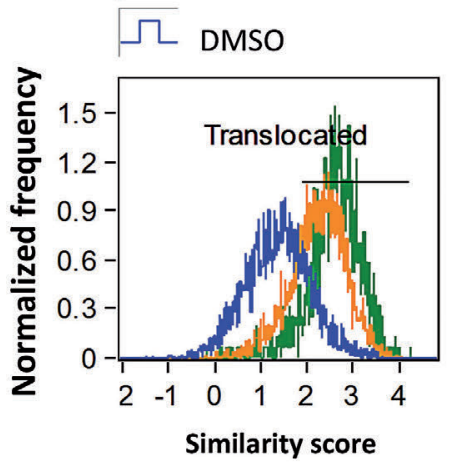

E

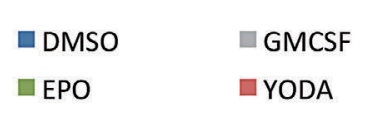

-YODA+GMCSF $\square$ YODA+EGTA

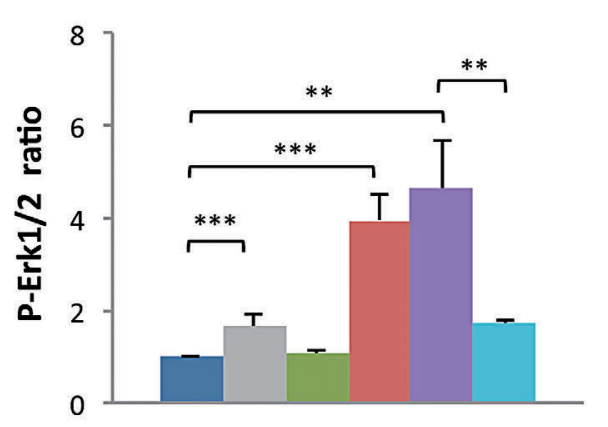

H
C

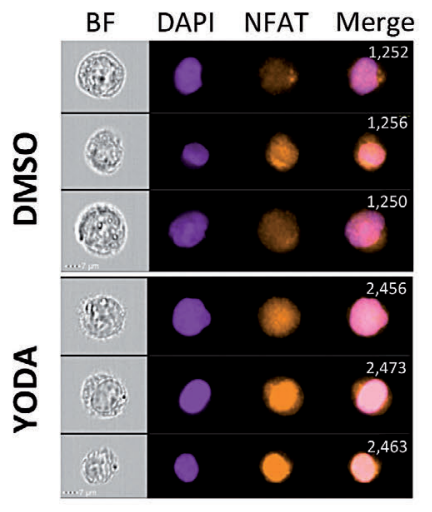

$\mathbf{F}$

DMSO $\square$ YODA
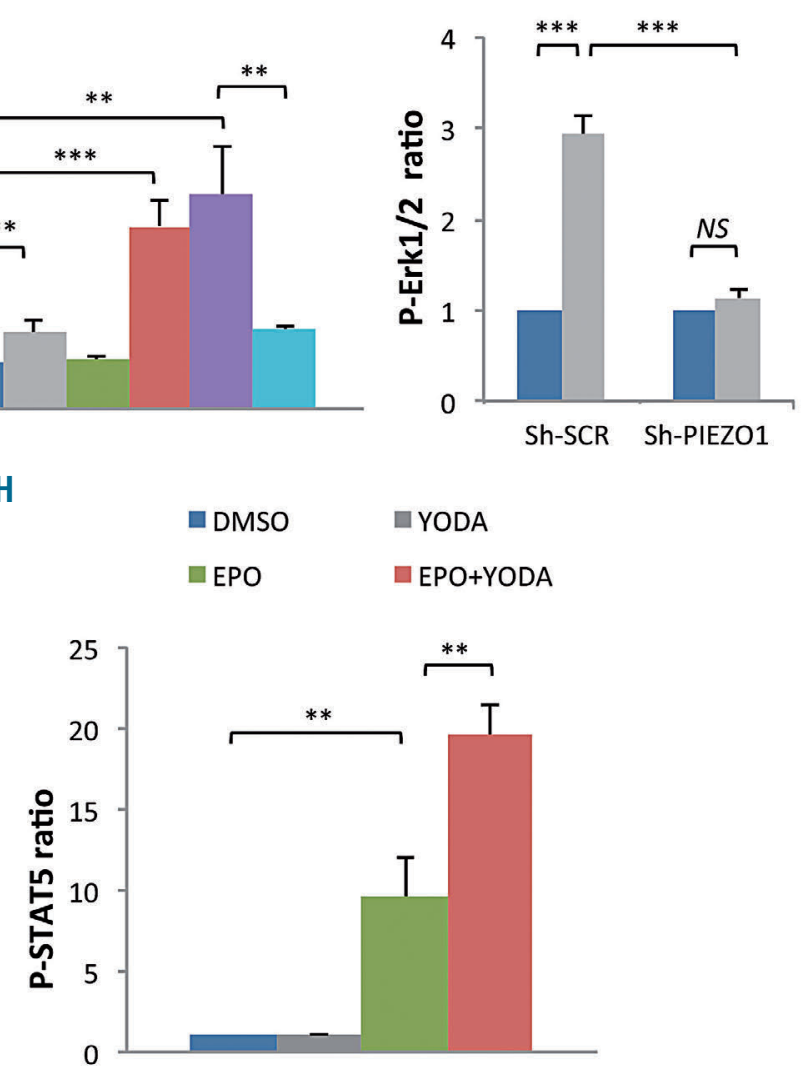

Figure 6. YODA1 activates NFAT, ERK and STAT5 pathways in erythroid cells. (A) The decrease in glycophorin A (GPA) in UT7/EPO cells after exposure to 5 MM YODA1 was blocked by concomitant exposure to $5 \mathrm{uM}$ tacrolimus (32+8\% vs. $70 \pm 2.5 \%$ ). (B) NFAT nuclear translocation secondary to exposure to 10 uM YODA1, assessed on ImageStream ${ }^{\circledR X}$ by the similarity score (SS) value in UT7/EPO cells, after overnight starvation of serum and erythropoietin (EPO). The SS is a mathematical tool used in Amnis IDEAS ${ }^{\circledR}$ software to assess the co-localization of a fluorescent signal (NFATc1-PE) and 4',6-diamidino-2-phenylindole (DAPI) nuclear staining. A high SS value means a highly translocated state. (C) Images of NFATc1 cellular localization using live imaging flow cytometry. After exposure to dimethylsulfoxide (DMSO), NFATc1 was preferentially localized in the cytosol, whereas 10 uM YODA1 increased NFATc1 nuclear translocation. Images were extracted from Amnis IDEAS ${ }^{\circledast}$ software for mean SS values of each condition. (D) In UT7/GM cells, $10 \mu \mathrm{M}$ U0126 induced high glycophorin A (GPA) expression (94 $\pm 0.2 \%$ ) compared to that following exposure to DMSO (18 $\pm 2 \%$ ) in medium containing granulocyte-monocyte colony-stimulating factor (GMCSF), and reverted the YODA1-mediated GPA repression when EPO was added (86 $\pm 1 \%$ ). Cells were incubated with U0126 for 30 min before stimulation with $5 \mu \mathrm{M}$ YODA1, then cultured for $72 \mathrm{~h}$. (E) ERK phosphorylation assessed by PhosphoFlow in UT7/GM cells. Values shown are the $\mathrm{p}$-ERK ratio relative to DMSO alone. GMCSF induced mild ERK phosphorylation ( $\times 1.65 \pm 0.27)$ whereas $10 \mathrm{U} / \mathrm{mL} E P O$ did not ( $\times 1.06 \pm 0.1$, $P=N S$ ). YODA1 (10 $\mu \mathrm{M})$ induced strong ERK phosphorylation (x3.96 \pm 0.581 ), an effect that was markedly inhibited by 2 mM ethylene glycol tetra-acetic acid (EGTA) ( $\times 1.71 \pm 0.05)$. (F) ERK phosphorylation assessed by PhosphoFlow in UT7/EPO cells. Sh-RNA-mediated PIEZO1 knockdown inhibited the $10 \mu \mathrm{M}$ YODA1-induced ERK phosphorylation (fold P-ERK increase in Sh-SCR-transduced cells: $x 2.93 \pm 0.2$; in Sh-PIEZO1-transduced cells: $x 1.13 \pm 0.1$ ). (G) ERK phosphorylation assessed by PhosphoFlow in primary human CD34+derived erythroid cells. YODA1 did not induce ERK phosphorylation ( $x 1.04 \pm 0.16, P=N S$ ), whereas $5 \mathrm{U} / \mathrm{mL}$ EPO did moderately ( $1.49 \pm 0.4)$. YODA1 synergized with EPO to induce ERK phosphorylation (x3.21 \pm 0.62 ). (H) STAT5 phosphorylation assessed by PhosphoFlow in primary human CD34+-derived erythroid cells. Compared to DMSO, YODA1 did not induce STAT5 phosphorylation ( $x 1.03 \pm 0.09, P=N S)$, whereas EPO did $(x 9.6 \pm 2.4)$, and YODA1 enhanced EPO-driven STAT5 phosphorylation ( $x 19.6 \pm 1.9)$. $(\mathrm{n}=3$ in $\mathrm{A}$ and $\mathrm{D}$, and $\mathrm{n}=4$ in all other experiments); $* * * P<0.001 ; * * P<0.01 ; * P<0.05$ 
Figures S8 and S9. Cultures of cells from four patients could be driven beyond day 10 and showed progressive terminal maturation and enucleation, but at a heterogeneous rate, confirming that erythroid differentiation was delayed but not totally blocked (Online Supplementary Figure S10).

\section{Discussion}

The mechanotransductor PIEZO1 has a well-described role in regulating hydration and volume of mature erythrocytes. ${ }^{12,28}$ Indeed, activating mutations are responsible for most HX cases and, recently, a frequent polymorphism (E756del) has been associated with resistance to malaria in African populations, ${ }^{29,30}$ although its influence on red cell hydration status is still controversial. ${ }^{31}$ PIEZO1 expression during erythropoiesis has been previously studied using RNA-sequencing analysis, the results of which were in agreement with our data that PIEZO1 is expressed in early progenitors ${ }^{14}$ and decreases during terminal maturation. ${ }^{23}$ These findings were confirmed at a protein level by extensive proteomic analyses of human erythroid differentiation. ${ }^{24}$ Whether PIEZO1 has a specific role during erythropoiesis is not known, although evoked in recent case reports. ${ }^{19,32}$ Our data show for the first time that PIEZO1 is expressed and functional in erythroid progenitors, and that its activation influences erythroid differentiation, both in leukemic cell lines and primary erythroid cells. Indeed, PIEZO1 activation maintained cells at an immature GPA low stage for longer and tilted the transcriptional balance in favor of genes associated with an immature stage, such as GATA2 and BMI1, at the expense of genes associated with terminal differentiation, such as GATA1, GPA, ALAS2 or $\alpha$ and $\beta$ globin without myeloid bias. ${ }^{33,34}$ Of note, we used the chemical activator YODA1 to evaluate the effects of PIEZO1 on erythropoiesis. ${ }^{25}$ The phenotype was dependent on PIEZO1 since it was abrogated after Sh-RNA mediated PIEZO1 knockdown, and was also confirmed in erythroid cells from HX patients carrying PIEZO1 gain-of-function mutations. Interestingly, we observed that PIEZO1 knockdown using a Sh-RNA strategy enhanced erythroid differentiation in UT7 cells. Since PIEZO1 activation was associated with an undifferentiated state in UT7 cells, we may assume that a low PIEZO1 level at the cell surface could lead to a lower "basal" activation rate and favor cell differentiation at the expense of cell proliferation. Of note, it was intriguing that a $50 \%$ reduction of PIEZO1 expression was sufficient to revert the YODA1-mediated effects. Although the GFP intensity determined the strength of YODA1 blockade, Sh-PIEZO1 significantly - but not totally - blocked the effects of YODA1 in GFP intermediate cells. This may argue for a threshold effect of PIEZO1, which could be further confirmed in patch-clamp experiments.

PIEZO1 is known as a non-selective cation channel. ${ }^{7}$ We showed that the effect of PIEZO1 activation on erythropoiesis was $\mathrm{Ca}^{2+}$-dependent, as described in mature red cells as well as in many other 'PIEZO1-sensitive' cells such as endothelial, urothelial, and epithelial cells., ${ }^{5,15}$ However, the consequences of a PIEZO1-mediated increase in intracellular $\mathrm{Ca}^{2+}$ may depend on the cell type. In mature red cells, the observed phenotype (i.e., red cell dehydration) occurs because of a secondary activation of the Gardos channel, which in turn exports $\mathrm{K}^{+}$and induces loss of water. ${ }^{13}$ The effects of PIEZO1 on erythropoiesis seem not to occur through this pathway, since the Gardos inhibitor Senicapoc could not revert the phenotype. $\mathrm{Ca}^{2+}$ influx has previously been shown to be involved during erythropoiesis. Notably, it has been suggested that EPO

Table 1. Characteristics of the 14 patients, 11 families and 10 PIEZO1 mutations, number of patients and family per mutation and main hematologic features.

\begin{tabular}{|c|c|c|c|c|c|c|c|c|c|c|c|c|}
\hline $\begin{array}{l}\text { Mutation } \\
\text { Id }\end{array}$ & $\begin{array}{l}\text { Patient } n \text { / } \\
\text { Family n }\end{array}$ & Exon & $\begin{array}{c}\text { PIEZ01 mutation } \\
\text { cDNA }\end{array}$ & Protein & Age & $\begin{array}{l}\text { Hb } \\
\text { (g/L) }\end{array}$ & $\begin{array}{l}\text { MCV } \\
\text { (fiL) }\end{array}$ & $\begin{array}{c}\text { Reticulocytes } \\
\times 10^{\circ} / \mathrm{L}\end{array}$ & $\begin{array}{l}\text { Ferritin } \\
(\mu g / L)\end{array}$ & $\begin{array}{c}\text { MRI } \\
\text { (umol/g) }\end{array}$ & $\begin{array}{c}\text { N. of } \\
\text { experiments }\end{array}$ & $\begin{array}{l}\text { Erythroid } \\
\text { s phenotype }\end{array}$ \\
\hline HX\#1 & $1 / 1$ & 14 & c. $1792 \mathrm{G}>\mathrm{A}$ & p.Val598Met & 43 & 144 & 107 & 404 & 828 & 190 & $\mathrm{~N}=3$ & High \\
\hline HX\#2 & $2 / 2$ & $\begin{array}{l}18 \\
18\end{array}$ & $\begin{array}{l}\text { c. } 2344 \mathrm{G}>\mathrm{A} \\
\text { c. } 2423 \mathrm{G}>\mathrm{A}\end{array}$ & $\begin{array}{l}\text { p.Gly782Ser } \\
\text { p.Arg808Gln }\end{array}$ & 55 & 142 & 101 & 441 & 613 & 95 & $\mathrm{~N}=3$ & High \\
\hline HX\#3 & $\begin{array}{l}3 / 3 \\
4 / 3\end{array}$ & $\begin{array}{l}42 \\
42\end{array}$ & $\begin{array}{l}\text { c. } 6058 \mathrm{G}>\mathrm{A}^{*} \\
\text { c. } 6058 \mathrm{G}>\mathrm{A}^{*}\end{array}$ & $\begin{array}{l}\text { p.Ala2020Thr } \\
\text { p.Ala2020Thr }\end{array}$ & $\begin{array}{l}31 \\
61\end{array}$ & $\begin{array}{l}146 \\
174\end{array}$ & $\begin{array}{l}85 \\
91\end{array}$ & $\begin{array}{l}173 \\
293\end{array}$ & $\begin{array}{c}30 \\
980\end{array}$ & $\begin{array}{l}\text { NA } \\
104\end{array}$ & $\begin{array}{l}\mathrm{N}=1 \\
\mathrm{~N}=1\end{array}$ & $\begin{array}{l}\text { Mild/moderate } \\
\text { Mild/moderate }\end{array}$ \\
\hline HX\#4 & $5 / 4$ & 42 & $\begin{array}{l}\text { c. } 6007 \mathrm{G}>\mathrm{A} \\
\text { c. } 7471 \mathrm{C}>\mathrm{T}\end{array}$ & $\begin{array}{l}\text { p.Ala2003Thr } \\
\text { p.Arg2491Trp }\end{array}$ & 24 & 118 & 99 & 451 & 86 & NA & $\mathrm{N}=3$ & High \\
\hline $\mathrm{HX \# 5}$ & $6 / 5$ & 51 & c.7391A >C & p.His2464Pro & 36 & 181 & 87 & 205 & 71 & NA & $\mathrm{N}=1$ & High \\
\hline $\mathrm{HX \# 6}$ & $7 / 6$ & 16 & $\begin{array}{c}\text { c. } 2152 \mathrm{G}>\mathrm{A} \\
\text { c. } 7463 \mathrm{G}>\mathrm{A \#}\end{array}$ & $\begin{array}{c}\text { p.Gly718Ser } \\
\text { p.Arg2488GIn }\end{array}$ & 51 & 152 & 93 & 167 & 119 & 34 & $\mathrm{~N}=1$ & Mild/moderate \\
\hline HX\#7 & $8 / 7$ & 51 & c. $7529 \mathrm{C}>\mathrm{T}$ & p.Pro2510Leu & 43 & 112 & 83 & 183 & NA & NA & $\mathrm{N}=1$ & High \\
\hline HX\#8 & $9 / 8$ & 16 & c. $2042 \mathrm{~T}>\mathrm{C}$ & p.Phe681Ser & 44 & 145 & 102 & 248 & 86 & 15 & $\mathrm{~N}=1$ & High \\
\hline $\mathrm{HX \# 9}$ & $10 / 9$ & 51 & c. $7367 \mathrm{G}>\mathrm{A}^{*}$ & p.Arg2456His & 63 & 112 & 120 & 288 & 535 & 200 & $\mathrm{~N}=2$ & High \\
\hline HX\#10 & $\begin{array}{l}11 / 10 \\
12 / 10 \\
13 / 10\end{array}$ & 16 & $\begin{array}{l}\text { c. } 2005 \mathrm{G}>\mathrm{T} \\
\text { c. } 2005 \mathrm{G}>\mathrm{T} \\
\text { c. } 2005 \mathrm{G}>\mathrm{T}\end{array}$ & $\begin{array}{l}\text { p. Asp669Tyr } \\
\text { p. Asp669Tyr } \\
\text { p. Asp669Tyr }\end{array}$ & $\begin{array}{l}62 \\
39 \\
35\end{array}$ & $\begin{array}{l}141 \\
142 \\
149\end{array}$ & $\begin{array}{c}98 \\
104 \\
107\end{array}$ & $\begin{array}{l}249 \\
496 \\
290\end{array}$ & $\begin{array}{l}1000 \\
889 \\
507\end{array}$ & $\begin{array}{l}330 \\
\text { NA } \\
180 \\
\end{array}$ & $\begin{array}{l}\mathrm{N}=1 \\
\mathrm{~N}=1 \\
\mathrm{~N}=1\end{array}$ & $\begin{array}{l}\text { Mild/ moderate } \\
\text { Mild/ moderate } \\
\text { Mild/ moderate }\end{array}$ \\
\hline HX\#11 & $14 / 11$ & 51 & c. $7367 \mathrm{G}>\mathrm{A}^{*}$ & p.Arg2456His & 78 & 129 & 85 & 221 & 600 & NA & $\mathrm{N}=1$ & High \\
\hline
\end{tabular}

*Functional studies showing slower PIEZO1 inactivation kinetics. ${ }^{8,12}$ \#: Functional studies showing a lower PIEZO1 activation threshold. ${ }^{8}$ Ferritin assays and magnetic resonance imaging evaluation of iron liver content were performed at diagnosis or before iron chelation/phlebotomy. Patients HX\#9 and HX\#11, from two different families, had the same PIEZO1 mutation. Id: identity, Hb: hemoglobin; MCV: mean corpuscular volume; MRI: magnetic resonance imaging. 
could indirectly regulate $\mathrm{Ca}^{2+}$ currents by modulating $\mathrm{Ca}^{2+}$ channel expression at the cell surface. ${ }^{35,36}$ However, consequences on erythroid maturation remain largely unknown. $\mathrm{Ca}^{2+}$ signaling is required during enucleation in mice, ${ }^{37}$ but we did not observe any effect on enucleation rate when PIEZO1 was activated in the last step of differentiation (data not shown). In contrast, we did observe that $\mathrm{Ca}^{2+}$ influx through PIEZO1 had an early effect on erythro- poiesis and noticed that NFAT, ERK1/2 and STAT5 were activated by PIEZO1-dependent $\mathrm{Ca}^{2+}$ entry in erythroid cells. In particular, we found that ERK was strongly phosphorylated by YODA1. ERK phosphorylation was inhibited after PIEZO1 knockdown, a result contrasting with a recent study showing that YODA1 phosphorylated ERK in endothelial cells in a PIEZO1-independent manner. ${ }^{38}$ However, this observation was made using chemical
A

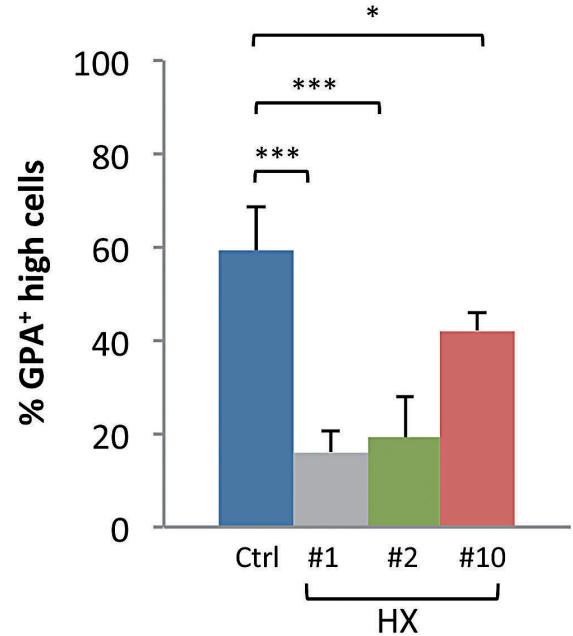

B

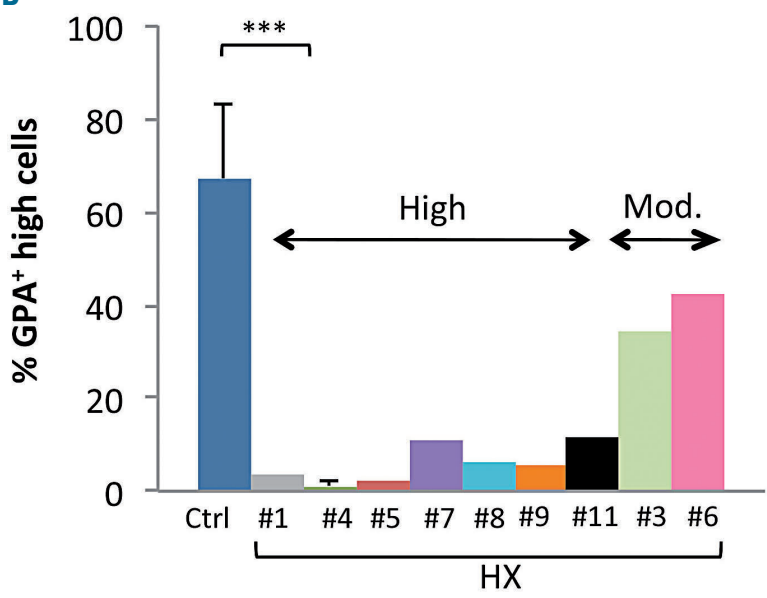

D
- Control $\quad$ HX\#1

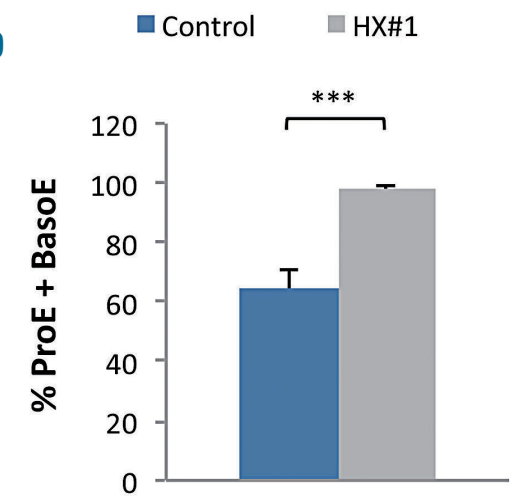

$\mathrm{E}$
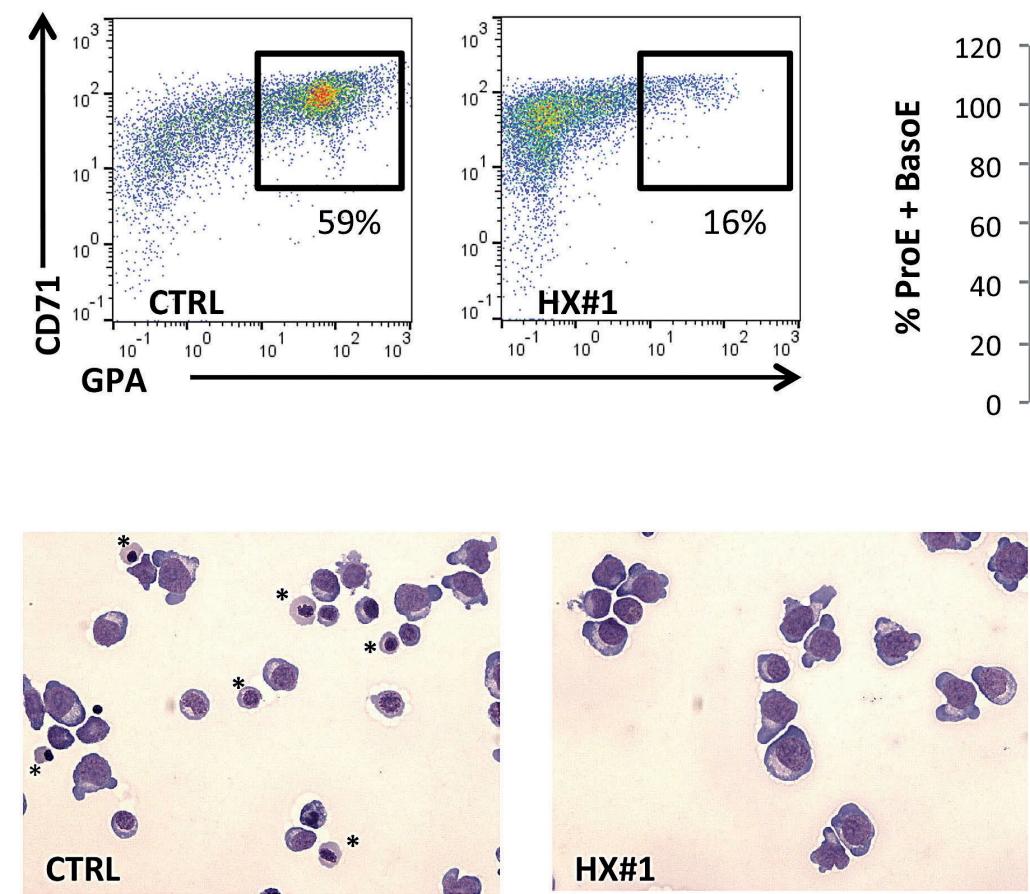

Figure 7. Delay in erythroid differentiation of progenitor cells obtained from patients with PIEZ01-mutated hereditary xerocytosis. (A) Culture of CD34 ${ }^{+}$-derived erythroid cells from patients with hereditary xerocytosis (HX); differentiation was assessed at day 10 by multiparametric flow cytometry (MFC). The mean percentage of CD $71^{*} / \mathrm{GPA}^{\text {High }}$ cells was $59 \pm 9 \%$ in control samples, $16 \pm 5 \%$ in $\mathrm{HX} \# 1(P<0.001), 19 \pm 9 \%$ in $\mathrm{HX} \# 2(P<0.01)$, and $42 \pm 4 \%$ in $\mathrm{HX} \# 10(\mathrm{P}<0.05)$. $(\mathrm{n}=5$ for control samples; $n=3$ for HX\#1, 2 and 10). (B) Culture of mononuclear cells from HX patients; differentiation assessed on day 10 by MFC ( $n=9$ for control samples; $n=3$ for HX\#4;

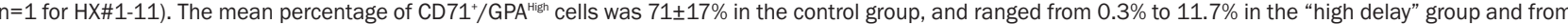
$22.5 \%$ to $44 \%$ in the "moderate delay" group. (C) Illustrative CD71/GPA MFC plots at day 10 of erythroid differentiation of CD34 cells obtained from patient HX\#1 (right) and from one representative control sample (left). (D) Cytological analysis after staining with May-Grünwald-Giemsa (MGG): count of immature erythroblasts [proerythroblasts (ProE) and basophilic erythroblasts (BasoE)] on day 10, x200 magnification. Immature erythroblasts were 64.2 $\pm 6.2 \%$ in control samples vs. $97.7 \pm 1.5 \%$ in $\mathrm{HX \# 1}$ ( $n=6$ control samples; $n=3$ for patient HX\#1). (E) Example of cytology on day 10 for patient HX\#1 (right) and for a control sample (left), MGG staining, $x 200$ magnification. * show orthochromatic erythroblasts. $* * * P<0.001 ; * * P<0.01 ; * P<0.05$. 
PIEZO1 inhibitors, which are much less specific than a ShRNA based strategy. The PIEZO1-Ca ${ }^{2+}$-ERK axis has been described in epithelial cells, in which it regulated the cell cycle and survival. ${ }^{15}$ Whether $\mathrm{Ca}^{2+}$ entry into erythroblasts through PIEZO1 was sufficient to activate the ERK pathway by itself or whether it triggered secondary pathways is under investigation. ERK1/2 activation has been shown to promote early human progenitor cell expansion and its constitutive activation in mice blocked differentiation of fetal liver erythroid progenitor cells..$^{39-41}$ Alternatively, ERK activation through EPO has been shown to promote erythroid differentiation in a MASL1-dependent manner. ${ }^{42}$ This discrepancy in the role of ERK1/2 in erythroid differentiation may depend on its activation level. Our results showed that PIEZO1 activation synergized both EPOdependent ERK and STAT5 phosphorylation in primary erythroblasts, suggesting that PIEZO1 may act as a modulator of EPO signaling during human erythropoiesis. Previous data suggested that erythroid regulation involved balanced STAT5 and ERK1/2 phosphorylation, and we assume that PIEZO1 may modify this equilibrium to delay erythroid terminal differentiation..$^{43}$ Of note, YODA1-sustained ERK1/2 activation in UT7/GM cells did not promote cell proliferation, in contrast to other cell types, but instead led to accumulation in the G0/G1 phase of the cell cycle. ${ }^{41,44}$ This may be due to the fact that YODA1 phosphorylated ERK1/2 but not STAT5 in UT7/GM cells in which proliferation was mainly dependent on STAT5. Alternatively, PIEZO1 activation could modulate cell cycle regulators as previously shown in canine epithelial cells. ${ }^{6}$ This highlights the heterogeneous role of PIEZO1 activation, which differs depending on the type of cell. We also showed that the effects of YODA1 required NFAT activation and nuclear translocation, which have been involved in mice erythropoiesis. ${ }^{45}$ Whether this pathway also depends on EPO signaling is under investigation.

Erythroid cells from HX patients carrying PIEZO1 gainof-function mutations had a similar phenotype as the one observed after PIEZO1 chemical activation. This highlights the pathophysiological relevance of this ion channel, proving that how PIEZO1 is activated (i.e., constitutive mutation vs. chemical activation) is not critical. Moreover, it suggests that the HX erythroid phenotype may involve a certain degree of impaired erythropoiesis, as evoked in two recent case reports, which may participate in the high rate of iron overload described in these patients., ${ }^{8,1922,32}$ However, the delay in erythroid differentiation does not translate into anemia since most patients have a totally compensated hemolysis. First, dyserythropoiesis may be mild in vivo. Second, it has been suggested that PIEZO1HX red blood cells have an increased hemoglobin affinity for oxygen. ${ }^{46}$ Therefore, hemoglobin level could represent a balance between factors that tend to decrease it (hemolysis, dyserythropoiesis) and factors that tend to increase it (hemoglobin hyperaffinity). We were unable to find a clear correlation between in vivo erythrocyte parameters in HX patients and the in vitro erythroid phenotype. This may be due to different mechanisms involved, since mature red cell dehydration is known to be Gardosdependent while the YODA1-induced erythroid delay was insensitive to Senicapoc. However, it would be of great interest to correlate, for each mutation, the intensity of the erythroid phenotype with functional tests such as high-throughput patch clamping on red cells. ${ }^{47}$ Alternatively, modeling the different HX mutations through viral transduction in normal erythroid progenitors would represent an interesting way to better understand the disease heterogeneity and to highlight the potential role of other genetic factors that may modulate the phenotype.

In summary, we describe here a role for PIEZO1 during human erythroid differentiation, in erythroleukemic cell lines, in normal primary erythroblasts after exposure to YODA1, and in primary cells from HX patients carrying an activating PIEZO1 mutation. We observed a delay in terminal erythroid maturation which depended on $\mathrm{Ca}^{2+}$ entry, and NFAT, STAT5 and ERK1/2 pathway activation. We observed that PIEZO1 could synergize with EPO-signaling during human erythropoiesis and that its constitutive activation in HX led to impairment in proliferation and differentiation of erythroid progenitors, highlighting a new pathophysiological mechanism in this rare disorder.

\section{Acknowledgments}

The authors are grateful to the University Hospital of Amiens (CHU Amiens), the Club du Globule Rouge et du Fer (CGRF), and the Réseau Hématologie Picardie (RHEPI) for their support. CHU Amiens provided financial support through the HEMASTEM project. We thank Prof. Stephane Giraudier (Paris, France) for the MEK-DN plasmid.

\section{References}

1. Coste B, Mathur J, Schmidt M, et al. Piezo1 and Piezo2 are essential components of distinct mechanically activated cation channels. Science. 2010;330(6000):55-60.

2. Saotome K, Murthy SE, Kefauver JM, Whitwam T, Patapoutian A, Ward AB. Structure of the mechanically activated ion channel Piezo1. Nature. 2018;554(7693):481486.

3. Zhao Q, Zhou H, Chi S, et al. Structure and mechanogating mechanism of the Piezo1 channel. Nature. 2018;554(7693):487-492

4. Cinar E, Zhou S, DeCourcey J, Wang Y, Waugh RE, Wan J. Piezo1 regulates mechanotransductive release of ATP from human RBCs. Proc Natl Acad Sci U S A. 2015;112(38):11783-11788.

5. Cahalan SM, Lukacs V, Ranade SS, Chien S, Bandell M, Patapoutian A. Piezo1 links mechanical forces to red blood cell volume. Elife. 2015;4

6. Wu J, Young M, Lewis AH, Martfeld AN, Kalmeta B, Grandl J. Inactivation of mechanically activated Piezo1 ion channels is determined by the C-terminal extracellular domain and the inner pore helix. Cell Rep. 2017;21(9):2357-2366

7. Gnanasambandam R, Bae C, Gottlieb PA, Sachs F. Ionic selectivity and permeation properties of human PIEZO1 channels. PLoS One. 2015;10(5):e0125503.

8. Andolfo I, Alper SL, De Franceschi L, et al.
Multiple clinical forms of dehydrated hereditary stomatocytosis arise from mutations in PIEZO1. Blood. 2013;121(19):3925-3935.

9. Zarychanski R, Schulz VP, Houston BL, et al Mutations in the mechanotransduction protein PIEZO1 are associated with hereditary xerocytosis. Blood. 2012;120(9):1908-1915.

10. Albuisson J, Murthy SE, Bandell M, et al. Dehydrated hereditary stomatocytosis linked to gain-of-function mutations in mechanically activated PIEZO1 ion channels. Nat Commun. 2013;4:1884.

11. Bae C, Gnanasambandam R, Nicolai C, Sachs F, Gottlieb PA. Xerocytosis is caused by mutations that alter the kinetics of the mechanosensitive channel PIEZO1. Proc Natl Acad Sci U S A. 2013;110(12):E11621168. 
12. Glogowska E, Schneider ER, Maksimova Y, et al. Novel mechanisms of PIEZO1 dysfunction in hereditary xerocytosis. Blood. 2017;130(16):1845-1856.

13. Rapetti-Mauss R, Picard V, Guitton C, et al. Red blood cell Gardos channel (KCNN4): the essential determinant of erythrocyte dehydration in hereditary xerocytosis. Haematologica. 2017;102(10):e415-e418.

14. Li J, Hale J, Bhagia P, et al. Isolation and transcriptome analyses of human erythroid progenitors: BFU-E and CFU-E. Blood. 2014;124 (24):3636-3645

15. Gudipaty SA, Lindblom J, Loftus PD, et al. Mechanical stretch triggers rapid epithelial cell division through Piezo1. Nature. 2017;543(7643):118-121.

16. Miyamoto T, Mochizuki T, Nakagomi H, et al. Functional role for Piezo1 in stretchevoked $\mathrm{Ca} 2+$ influx and ATP release in urothelial cell cultures. J Biol Chem. 2014; 289(23):16565-16575.

17. Li J, Hou B, Tumova S, et al. Piezo1 integration of vascular architecture with physiological force. Nature. 2014:515(7526):279-282.

18. Del Mármol JI, Touhara KK, Croft G, MacKinnon R. Piezo1 forms a slowly-inactivating mechanosensory channel in mouse embryonic stem cells. Elife. 2018;7

19. Park J, Jang W, Han E, et al. Hereditary dehydrated stomatocytosis with splicing site mutation of PIEZO1 mimicking myelodysplastic syndrome diagnosed by targeted next-generation sequencing. Pediatr Blood Cancer. 2018;65(7):e27053.

20. Garçon L, Lacout C, Svinartchouk F, et al. Gfi-1B plays a critical role in terminal differentiation of normal and transformed erythroid progenitor cells. Blood. 2005;105(4): 1448-1455.

21. Walrafen P, Verdier F, Kadri Z, Chrétien S, Lacombe C, Mayeux P. Both proteasomes and lysosomes degrade the activated erythropoietin receptor. Blood. 2005;105(2): 600-608.

22. Picard V, Guitton C, Thuret I, et al. Clinical and biological features in PIEZO1-hereditary xerocytosis and Gardos-channelopathy: a retrospective series of 126 patients. Haematologica. 2019;104(8):1554-1564.

23. An X, Schulz VP, Li J, et al. Global transcriptome analyses of human and murine terminal erythroid differentiation. Blood.
2014;123(22):3466-3477.

24. Gautier E-F, Ducamp S, Leduc M, et al. Comprehensive proteomic analysis of human erythropoiesis. Cell Rep. 2016;16(5): 1470-1484.

25. Syeda R, Xu J, Dubin AE, et al. Chemical activation of the mechanotransduction channel Piezo1. Elife. 2015;4.

26. $\mathrm{Hu}$ J, Liu J, Xue F, et al. Isolation and functional characterization of human erythroblasts at distinct stages: implications for understanding of normal and disordered erythropoiesis in vivo. Blood. 2013;121(16): 3246-3253.

27. Hogan PG. Transcriptional regulation by calcium, calcineurin, and NFAT. Genes Dev. 2003;17(18):2205-2232.

28. Gallagher PG. Disorders of erythrocyte hydration. Blood. 2017;130(25):2699-2708.

29. Caulier A, Rapetti-Mauss R, Guizouarn H Picard V, Garçon L, Badens C. Primary red cell hydration disorders: pathogenesis and diagnosis. Int J Lab Hematol. 2018;40 (Suppl 1):68-73.

30. Ma S, Cahalan S, LaMonte G, et al Common PIEZO1 allele in African populations causes RBC dehydration and attenuates Plasmodium infection. Cell. 2018;173 (2):443-455.e12.

31. Rooks H, Brewin J, Gardner K, et al. A gain of function variant in PIEZO1 (E756del) and sickle cell disease. Haematologica. 2019;104 (3):e91-e93.

32. Paessler M, Hartung H. Dehydrated hereditary stomatocytosis masquerading as MDS. Blood. 2015;125(11):1841.

33. Moriguchi T, Yamamoto M. A regulatory network governing Gata1 and Gata2 gene transcription orchestrates erythroid lineage differentiation. Int J Hematol. 2014;100(5): 417-424.

34. Hattangadi SM, Wong P, Zhang L, Flygare J, Lodish HF. From stem cell to red cell: regulation of erythropoiesis at multiple levels by multiple proteins, RNAs, and chromatin modifications. Blood. 2011;118(24):62586268

35. Cheung JY, Zhang XO, Bokvist K, Tillotson DL, Miller BA. Modulation of calcium channels in human erythroblasts by erythropoietin. Blood. 1997;89(1):92-100.

36. Chu X, Cheung JY, Barber DL, et al. Erythropoietin modulates calcium influx through TRPC2. J Biol Chem. 2002;277(37):
34375-34382

37. Wölwer CB, Pase LB, Russell SM, Humbert PO. Calcium signaling is required for erythroid enucleation. PLoS ONE. 2016;11(1): e0146201.

38. Dela Paz NG, Frangos JA. Yoda1-induced phosphorylation of Akt and ERK1/2 does not require Piezo1 activation. Biochem Biophys Res Commun. 2018;497(1):220-225.

39. Sui X, Krantz SB, You M, Zhao Z Synergistic activation of MAP kinase (ERK1/2) by erythropoietin and stem cell factor is essential for expanded erythropoiesis. Blood. 1998;92(4):1142-1149.

40. Zhang J, Socolovsky M, Gross AW, Lodish HF. Role of Ras signaling in erythroid differentiation of mouse fetal liver cells: functional analysis by a flow cytometry-based novel culture system. Blood. 2003;102(12):39383946.

41. Zhang J, Lodish HF. Constitutive activation of the MEK/ERK pathway mediates all effects of oncogenic $\mathrm{H}$-ras expression in primary erythroid progenitors. Blood 2004:104(6):1679-1687.

42. Kumkhaek C, Aerbajinai W, Liu W, et al. MASL1 induces erythroid differentiation in human erythropoietin-dependent CD34+ cells through the Raf/MEK/ERK pathway. Blood. 2013;121(16):3216-3227.

43. Arcasoy MO, Jiang X. Co-operative signalling mechanisms required for erythroid precursor expansion in response to erythropoietin and stem cell factor. Br J Haematol. 2005;130(1):121-129.

44. Marshall CJ. Specificity of receptor tyrosine kinase signaling: transient versus sustained extracellular signal-regulated kinase activation. Cell. 1995;80(2):179-185

45. Giampaolo S, Wójcik G, Klein-Hessling S Serfling E, Patra AK. NFAT-mediated defects in erythropoiesis cause anemia in II2-/- mice. Oncotarget. 2018;9(11):9632-9644.

46. Kiger L, Guitton C, Ghazal K, et al. Physiopathology of hereditary xerocytosis: Piezo gain of function mutations impact hemoglobin oxygen. Haematologica. 2017; 102(s2):446.

47. Rotordam GM, Fermo E, Becker N, et al. A novel gain-of-function mutation of Piezo1 is functionally affirmed in red blood cells by high-throughput patch clamp Haematologica. 2019;104(5):e179-e183. 